\title{
MoRic8 Is a Novel Component of G-Protein Signaling During Plant Infection by the Rice Blast Fungus Magnaporthe oryzae
}

\author{
Ya Li, ${ }^{1}$ Xia Yan, ${ }^{1}$ Hong Wang, ${ }^{1}$ Shen Liang, ${ }^{1}$ Wei-Bin Ma, ${ }^{1}$ Min-Yan Fang, ${ }^{1}$ Nicholas J. Talbot, ${ }^{2}$ and \\ Zheng-Yi Wang ${ }^{1,3}$ \\ ${ }^{1}$ State Key Laboratory for Rice Biology, Biotechnology Institute, Zhejiang University, Huajiachi Campus, Hangzhou, 310029, \\ People's Republic of China; ${ }^{2}$ School of Biosciences, University of Exeter, Geoffrey Pope Building, Exeter EX4 4QD, U.K. ; \\ ${ }^{3}$ Key Laboratory of Molecular Biology of Crop Pathogens and Insects, Ministry of Agriculture, Zhejiang University, \\ People's Republic of China
}

Submitted 15 June 2009. Accepted 30 September 2009.

\begin{abstract}
An insertional mutagenesis screen was used to investigate the biology of plant infection by the devastating rice blast pathogen, Magnaporthe oryzae. Here, we report the identification of a new mutant, LY-130, which is defective in multiple steps during infection-related morphogenesis and pathogenicity. Analysis of the mutation confirmed an insertion into gene MoRIC8, which encodes a 480-aminoacid protein that is a putative homologue of the Ric8 regulator of GTP-binding protein (G-protein) signaling, previously described in animals. Targeted gene deletion mutants of MoRIC8 were nonpathogenic and impaired in cellular differentiation associated with sporulation, sexual development, and plant infection. MoRic8 physically interacts with the $G \alpha$ subunit MagB in yeast two-hybrid assays and appears to act upstream of the cyclic AMP response pathway that is necessary for appressorium morphogenesis. Taken together, our results indicate that MoRic8 may act as a novel regulator of the G-protein signaling during infection-related development of rice blast fungus M. oryzae.
\end{abstract}

Rice blast, caused by Magnaporthe oryzae, is one of the most severe diseases of cultivated rice throughout the world. The fungus infects its host by means of a specialized infection structure known as an appressorium, which is a dome-shaped, melanin-pigmented cell. Once cuticle penetration has occurred, the fungus grows as bulbous primary infection hyphae within infected host cells. Recently, cytological evidence has demonstrated that invasive hyphae of $M$. oryzae are surrounded by the invaginated plant plasma membrane and move from cell to cell by means of plasmodesmata (Kankanala et al. 2007; Wilson and Talbot 2009). Due to its economic importance and experimental tractability, $M$. oryzae has recently emerged as a model organism for the study of plant diseases caused by fungi (Talbot 2003; Ebbole 2007; Wilson and Talbot 2009).

In $M$. oryzae, infection-related morphogenesis is controlled by the cyclic adenosine $3^{\prime}, 5^{\prime}$-cyclophosphate (cAMP) response pathway and activation of the Pmk1 mitogen-activated protein kinase (MAPK) cascade (Xu and Hamer 1996; Choi and Dean 1997; Caracuel-Rios and Talbot 2007). Initiation of M. oryzae appressorium formation requires cAMP signaling, because de-

Corresponding author: Z. Wang; E-mail: zhywang@zju.edu.cn letion of the MACl gene, which encodes adenylate cyclase, results in mutants which cannot form appressoria (Choi and

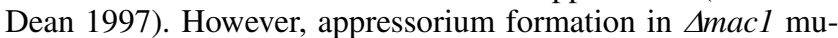
tants can be complemented by adding exogenous cAMP or by a second-site mutation in $S U M 1$, which encodes the regulatory subunit of cAMP-dependent protein kinase A (PKA), resulting in detachment and constitutive activation of the catalytic PKA subunit (Adachi and Hamer 1998). Consistent with this, gene replacement mutants of $C P K A$, which encodes the catalytic subunit of PKA, are delayed in appressorium formation and form small, misshapen, and nonfunctional appressoria (Mitchell and Dean 1995; Xu et al. 1997). The role of the M. oryzae Pmk1 MAPK pathway in regulating appressorium development has also been clearly established (Zhao et al. 2005; Park et al. 2006; Zhao and Xu 2007; Caracuel-Rios and Talbot 2007). In M. oryzae, $\Delta p m k 1$ mutants are nonpathogenic and fail to form appressoria, although mutants still respond to hydrophobic surfaces or exogenous cAMP by producing swollen germ tube tips (Xu and Hamer 1996). Activation of the Pmk1 MAPK occurs through the Mst7 and Mst11 proteins, which are homologous to the Saccharomyces cerevisiae Ste7 MAPK/ERK kinase (MAPKK) and the Ste11 MEK kinase (MAPKKK), respectively (Zhao et al. 2005). Mst50, a homologue of Ste50 from $S$. cerevisiae, acts as the adaptor or scaffold protein for the Mst11-Mst7 signaling module by directly interacting with both the Mst11 and Mst7 proteins (Park et al. 2006). Consistent with its role as an essential scaffold of the Pmk1 MAPK signaling pathway, deletion of MST50 leads to defects in appressorium formation and loss of pathogenicity (Park et al. 2006). Therefore, the cAMP pathway and Pmk1 MAPK cascade are essential regulators of appressorium development and pathogenicity in the rice blast fungus.

Heterotrimeric GTP-binding proteins (G-proteins) are known to have universal roles as signaling proteins in eukaryotic cells. In $M$. oryzae, upstream activation of both adenylate cyclase and Pmk1 MAPK appears to be mediated by G-proteins in response to physical characteristics and chemical signals released from the rice leaf surface. Three $M$. oryzae $\mathrm{G} \alpha$ subunits, MagA, MagB, and MagC, have been identified and functionally characterized (Liu and Dean 1997). Previous studies have shown that deletion of MAGA has no effect on vegetative growth, conidiation, or appressorium formation, and deletion of $M A G C$ reduces conidiation but did not affect vegetative growth or appressorium formation (Liu and Dean 1997). However, disruption of $M A G B$ significantly reduced vegetative 
growth, conidiation, appressorium formation, and pathogenicity (Liu and Dean 1997). MagB may sense surface cues and stimulate cAMP synthesis, because expression of a dominant active allele of $M A G B$ causes appressoria to form on noninductive hard surfaces, while addition of cAMP activates appressorium formation in a $\triangle m a g B$ mutant (Liu and Dean 1997; Fang and Dean 2000; Ebbole 2007). MGB1 encodes a $M$. oryzae $\mathrm{G} \beta$ subunit protein, which is the only $\mathrm{G} \beta$ protein encoded by the $M$. oryzae genome (Dean et al. 2005). Deletion of $M G B 1$ results in reduction of conidiation, defects in appressorium formation, and an inability to penetrate or grow invasively in rice leaves (Nishimura et al. 2003). However, similar to $\triangle m a g B$ mutants, appressoria could be partially induced by adding exogenous cAMP in $\Delta m g b l$ mutants, although these appressoria are abnormal in shape and cannot penetrate the rice surface cuticle (Nishimura et al. 2003). Therefore, Mgb1 may be involved in cAMP signaling regulating conidiation, surface recognition, and appressorium formation (Nishimura et al. 2003). A recent report has demonstrated that a regulator of G-protein signaling, Rgs1, interacts with all three of the Ga subunit proteins in M. oryzae (Liu et al. 2007); $\Delta r g s 1$ mutants are derepressed for appressorium development on hydrophilic surfaces and, thus, presumably negatively regulate appressorium formation when conditions are not appropriate, although a hard surface is still required (Liu et al. 2007) to allow their formation even in the absence of Rgs1. The identification of regulators will be helpful to allow better understanding of the Gprotein-mediated signaling in regulating early events during plant infection by the rice blast fungus.

In the last decade, Agrobacterium tumefaciens-mediated transformation (ATMT) has been developed as a large-scale gene tagging method in M. oryzae (Choi et al. 2007; Gupta and Chattoo 2007); Jeon et al. 2007; Meng et al. 2007). We have carried out a large-scale ATMT screen of M. oryzae to identify new genes that are necessary for infection-related morphogenesis and pathogenicity, and a mutant library containing more than 5,000 hygromycin-resistant transformants of $M$. oryzae has been generated. In this report, we present the identification and functional characterization of a novel pathogenicity-related gene of M. oryzae, MoRIC8, which is a homolog of the RIC8/RIC8A gene previously described in animals (Miller et al. 1996, 2000; Tall et al. 2003; Afshar et al. 2004; Siderovski and Willard 2005; Wang et al. 2005). Deletion of the MoRIC8 results in an inability to form appressoria on inductive surfaces. The defect of appressorium formation of $\Delta$ moric8 mutants could be partially restored by adding exogenous cAMP, although induced appressoria are still nonfunctional. MoRIC8 was expressed in cytoplasm of vegetative hyphae and conidia and was especially highly expressed in appressoria. Interestingly, MoRic8 interacts with the $\mathrm{G} \alpha$ subunit $\mathrm{MagB}$ in a yeast two-hybrid ( $\mathrm{Y} 2 \mathrm{H})$ assay, indicating that MoRic8 may act as a regulator of G-protein signaling in $M$. oryzae regulating multiple stages of infection-related morphogenesis in $M$. oryzae that are necessary for rice blast disease.

\section{RESULTS}

Identification of a nonpathogenic M. oryzae mutant LY-130.

To obtain pathogenicity-defective mutants, we carried out ATMT of the wild-type M. oryzae Guy11 (Leung et al. 1988) using a binary vector. In total, 5,248 hygromycin-resistant transformants of $M$. oryzae were generated using this approach. The blast-susceptible barley cv. Golden Promise was used for the first-round assessment using a barley cut-leaf assay. A rice cutleaf assay and rice seedling spray-inoculation assay were subsequently used to verify mutants showing an impairment in pathogenesis. In these screening experiments, seven mutants impaired in development of blast symptoms on the susceptible hosts were obtained. One of the mutants, LY-130 (Table 1), was identified as a nonpathogenic mutant, which was completely incapable of causing blast disease in susceptible barley and rice leaves, even when leaf surfaces were wounded by abrasion before inoculation (Fig. 1A and B). In order to determine whether the nonpathogenic phenotype was co-segregated with hygromycin resistance, LY-130 was crossed with the MAT1-1 strain TH3. However, in the vast majority of cases, no perithecia were observed at the junction between the two strains after 4 weeks of incubation, whereas control crosses between Guy11 and TH3 formed perithecia normally. To determine the T-DNA integration copies in LY-130, genomic DNA was digested with SalI, EcoRI, or $B a m \mathrm{HI}$ and then probed with the 1.4-kb hygromycin cassette. The DNA gel blot results showed that a single T-DNA integration event had occurred in the genome of LY-130 (Fig. 1C). Therefore, we decided to carry out further characterization of the LY-130 mutant.

\section{Identification of the T-DNA-tagged gene MoRIC8 in LY-130.}

In order to identify the T-DNA integration site in the LY-130 mutant, a $0.85-\mathrm{kb}$ genomic DNA flanking right site of the integration T-DNA was obtained using a high-efficiency thermal asymmetric interlaced (hiTAIL) polymerase chain reaction (PCR) strategy (Liu and Chen 2007), and sequenced. The TDNA insertion was found in the third exon of a predicted gene MGG_14008.6 (GenBank XP_001405357) located on supercontig 10 of chromosome $\mathrm{V}$. The exact integration site was at position $475750-$, which is 1,111 bp downstream of the predicted translational start site of the gene (Fig. 1D). We named the T-DNA-tagged gene MoRIC8 (M. oryzae RIC8) because it encodes a protein with similarity to Ric8/Ric8A proteins described previously in animals. However, homologues of Ric8/ Ric8A have not been previously characterized in phytopathogenic fungi.

To confirm the position and size of the introns of MoRIC8, reverse-transcription (RT)-PCR was carried out and the cDNA sequence and genome sequence compared. This confirmed that MoRIC8 has an open reading frame (ORF) of 1,867 bp interrupted by four introns $(63,59,64$, and $238 \mathrm{bp}$, respectively) and putatively encodes a 480 -amino-acid protein, which is $100 \%$

Table 1. Recombinant strains of Magnaporthe oryzae used in this study

\begin{tabular}{lll}
\hline Strain & \multicolumn{1}{c}{ Brief description } \\
\hline Guy11 & Wild-type, MAT1-2 & Leung et al. 1988 \\
LY-130 & T-DNA insertional mutant & This study \\
Q-3 & Amoric8 mutant of Guy11 & This study \\
Q-10 & Amoric8 mutant of Guy11 & This study \\
Q-8 & Ectopic transformant of Guy11 with integration of pRIC-KO \\
Q-11 & Ectopic transformant of Guy11 with integration of pRIC-KO \\
LY-130-R1 & LY-130 transformed with pRIC-GFP & This study \\
LY-130-R4 & LY-130 transformed with pRIC-GFP & This study \\
Q-10C & Q-10 transformed with pRIC-GFP & This study \\
Q-10C3 & Q-10 transformed with pRIC-GFP & This study \\
\hline
\end{tabular}


identical to the protein sequence predicted by automated annotation of the $M$. oryzae genome sequence (ID: MGG_14008.6; Broad Institute) (Fig. 1D).

In order to understand the nature of the T-DNA insertion event, a 0.63-kb PCR product, including genomic DNA flanking the left site of the integration, was amplified with the prim- ers TDNA-LB and MRC-LB (Table 2), and sequenced. Analysis of the sequence of the T-DNA insertion site and both right and left borders demonstrated that a 2.2-kb T-DNA sequence was integrated into the gene MoRIC8 and one base of guanine $(\mathrm{G})$ was deleted at the junction by the T-DNA integration (Fig. 1E).

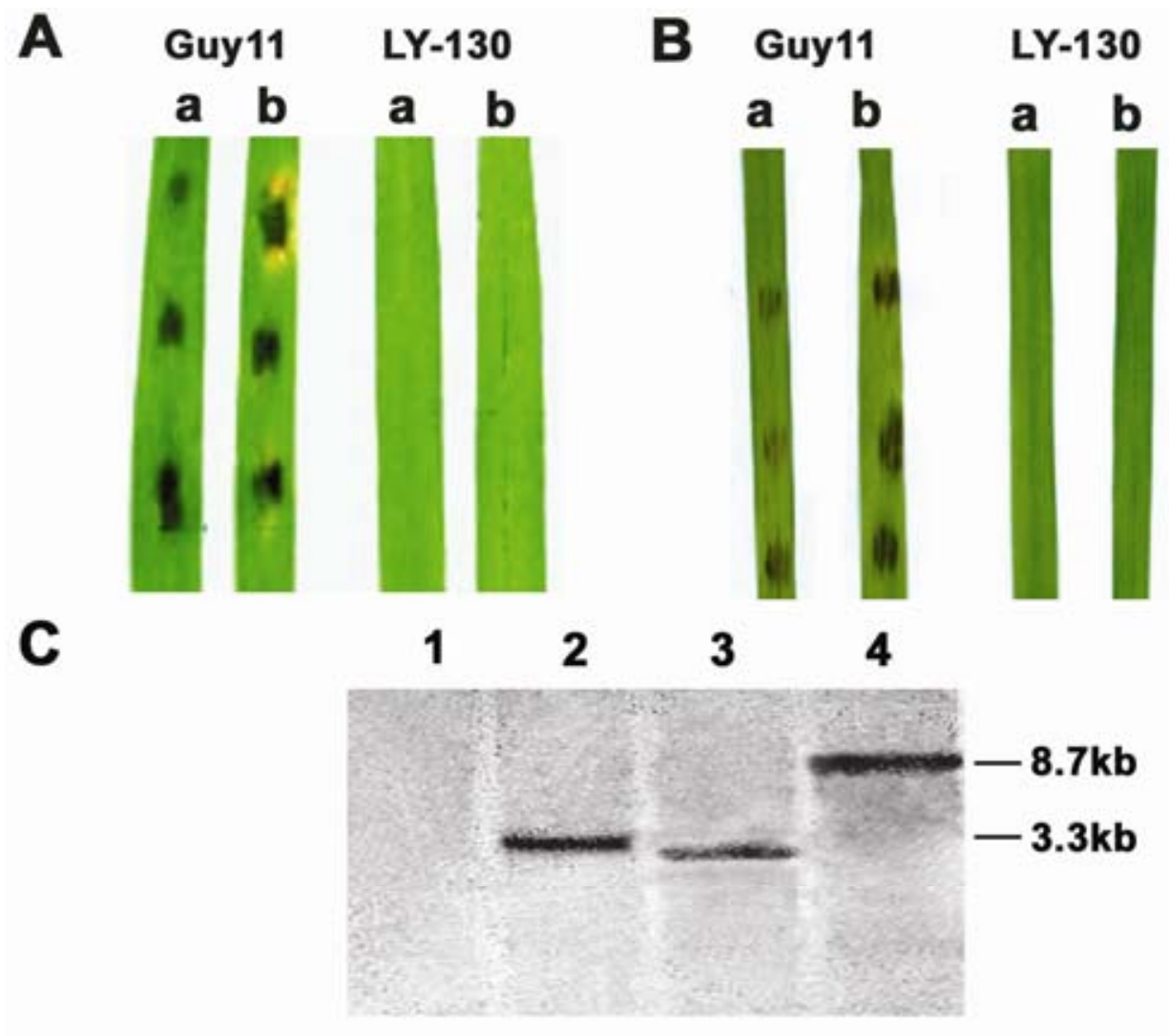

D

Genomic DNA(Supercontig 10)

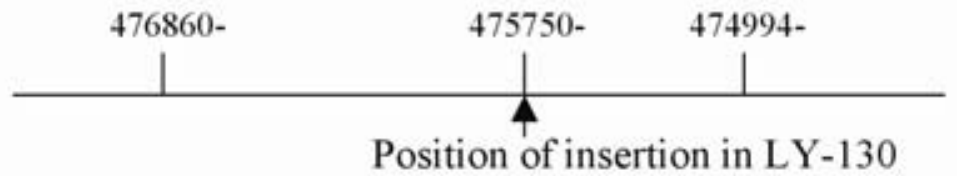

MoRIC 8 cDNA (this study; MGG_14008.6 Broad Inst.)

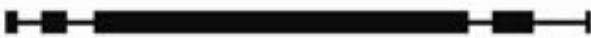

$0.5 \mathrm{~kb}$

E

RB

$2.2 \mathrm{~kb}$

LB

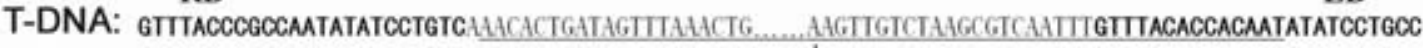

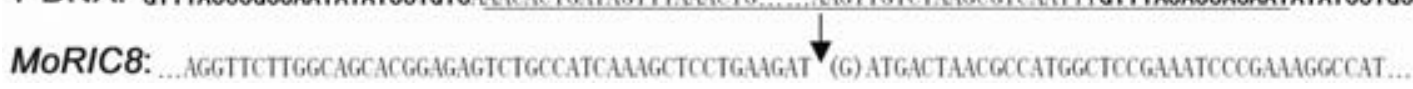

Fig. 1. Pathogenicity test of LY-130 mutant by a cut-leaf assay and integration of exogenous T-DNA in the mutant. A and B, Barley and rice leaf segments were inoculated with the conidial drops $\left(10 \mu \mathrm{l}\right.$ per drop) at a concentration $1 \times 10^{5}$ conidia $\mathrm{ml}^{-1}$ from Guy 11 and $\mathrm{LY}-130 ; \mathrm{a}=\mathrm{unwounded}$ leaf and $\mathrm{b}=\mathrm{abraded}$ leaf. $\mathbf{C}$, Southern blotting showing that a single T-DNA integration event occurred in the genome of LY-130. Lane 1, Guy11 genomic DNA was digested with Sall; lanes 2 through 4, LY-130 genomic DNA was digested with SalI, EcoRI, and BamHI, respectively. The DNA gel was probed with a 1.4-kb hygromycin-resistance gene cassette. D, Position of the T-DNA insertion in LY-130 mutant and structure of the MoRIC8 genomic locus. An arrow indicates the T-DNA insertion position in the Magnaporthe genome. The thick boxes represent the coding regions and the thin line joining these coding regions indicates the position of the introns. Genomic DNA and cDNA of MoRIC 8 were amplified with the primers C-F and C-R using Guy11 genomic DNA and reverse transcription cDNA, respectively, as templates in this study. The two sequences are 100\% identical to the locus MGG_14008.6 form Magnaporthe database. E, The pattern of T-DNA integrated into the Magnaporthe genome. Bold letters indicate T-DNA border sequences (LB, left border; RB, right border). The integrated T-DNA sequence (2.2 kb) is underlined. An arrow indicates the T-DNA insertion position in MoRIC8. The nucleotide in the brackets is deleted by T-DNA integration. 
Putative homologs of $R I C 8 / R I C 8 A$ were found in the genomes of related fungal species (Fig. 2A). MoRic8 showed 49.0, 43.3, and $38.7 \%$ amino acid identity with Neurospora crassa NCU02788, Gibberella zeae FG01511, and Sclerotinia sclerotiorum SS1G_02787, respectively ( $E$ values of $4 \mathrm{e}-126$, 2e-94, and 4e-93, respectively). However, MoRic8 showed only 16.5 and $15.1 \%$ amino acid identity with Mus musculus Ric8A and Caenorhabditis elegans Ric8, respectively, indicating the evolution difference of Ric8/Ric8A between fungi and animals. Phylogenetic analysis showed that MoRic8 is most closely related to the G. zeae hypothetical protein FG01511 and N. crassa NCU02788 but is genetically distant with Ric8/Ric8A from animal species (Fig. 2B). Interestingly, no homolog of RIC8 exists in S. cerevisiae, protists, or plants.

\section{MoRIC8 is essential for Magnaporthe oryzae} to cause rice blast disease.

To verify the nonpathogenic phenotype of LY-130, we performed a targeted gene deletion of MoRIC8 using the genereplacement vector pRIC-KO (Fig. 3A). The gene replacement was analyzed by PCR amplification with primers $3 \mathrm{~F}$ and $4 \mathrm{R}$ (Table 2) from transformants and Southern blots (Fig. 3A). The resulting 4 moric8 null mutants, Q-3 and Q-10 (Table 1), were selected based on the absence of hybridization to the deleted fragment probe and the presence of a single copy of the $H P H$ cassette (Fig. 3B). One of the transformants resulting from ectopically integrated pRIC-KO, Q-8, was used as a control strain (Fig. 3B). The morphology of the colonies of 4 moric8 (Q-3 and Q-10), LY-130, and wild-type strain Guy11 is shown

Table 2. Primers used in this study

\begin{tabular}{|c|c|}
\hline Name & Sequence $\left(5^{\prime} \rightarrow 3^{\prime}\right)^{a}$ \\
\hline TDNA-LB & CСТАAАACCAAAATCCAGTAC \\
\hline MRC-LB & СТАСТTTTGCTCCACTTTCTTG \\
\hline $1 \mathrm{~F}$ & ATTCGGACCCTTTTCTTTGA \\
\hline $2 \mathrm{R}$ & CATCGCGTTGTAGCCCACTT \\
\hline $3 \mathrm{~F}$ & GCGGTACCAGCAGTTGAAAGTTTATGG \\
\hline $4 \mathrm{R}$ & GCGGTACCCTTTTGCTCCACTTTCTTGT \\
\hline HPH-Xh-F & 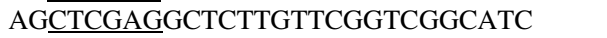 \\
\hline HPH-Sp-R & CGCGTACGTATTGAAGGAGCATTTTTGG \\
\hline HB-F & TAGGAAATGGCTGCTGAAGG \\
\hline HB-R & GCGGTACCCTTTTGCTCCACTTTCTTGT \\
\hline GFP-F & 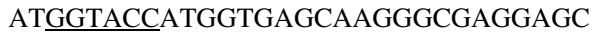 \\
\hline GFP-R & TAGGTACC $\overline{\text { GTGGAGATGTGGAGTGGGCGCTT }}$ \\
\hline $\mathrm{C}-\mathrm{F}$ & ATGTTGAATACCAATACACTGAAGG \\
\hline C-R & CTAAATTAGGCGGTACTTTTGCTC \\
\hline RIC-Q-F & GAACACCATGGAGGGTATGG \\
\hline RIC-Q-R & GAGGATCAAGGACGGATTCA \\
\hline MA-Q-F & AAGGCTACTCGGATGACGAA \\
\hline MA-Q-R & CGACCATGTAGTCGAGCAGA \\
\hline MB-Q-F & CGACCTCACCTACCGAATGT \\
\hline MB-Q-R & ATACGGTTGACCGTCTCGTC \\
\hline MC-Q-F & AAAGCACTATGGGTGGATGC \\
\hline MC-Q-R & AAACACGGTCTCGGTAATGC \\
\hline MB1-Q-F & GCCATTCAATTCTTCCCAGA \\
\hline MB1-Q-R & AGCCTGCCTGAAACTGATGT \\
\hline MG1-Q-F & GAGTATGGCCGATCTCAAGC \\
\hline MG1-Q-R & GGTACTGCTCCCCAGACAGA \\
\hline Tub-F & ACCCTCGCAACGGAAAG \\
\hline Tub-R & GACGAACTGGATGCTACGC \\
\hline RIC-E-F & GCGAATTCATGTTGAATACCAATACAC \\
\hline RIC-E-R & GTGAATTCCTAAATTAGGCGGTACTT \\
\hline MAGA-Nd-F & CATATGATGGGCGCTTGCATGA \\
\hline MAGA-Ps-R & CTGCAGTTACAGAATACCTGAGTCC \\
\hline MAGB-E-F & GAGAATTCATGGGTTGCGGAAT \\
\hline MAGB-B-R & GCGGATCCTTAGATCAGACCACAGA \\
\hline MAGC-E-F & GAATTCATGTGCTTCGGCAGCCGC \\
\hline MAGC-B-R & CGGGATCCTCACAAGATGAGTTGCTT \\
\hline
\end{tabular}

${ }^{\mathrm{a}}$ Introduced restriction sites are underlined. $K p n \mathrm{I}=$ GGTACC, $\mathrm{XhoI}=\mathrm{CT}$ CGAG, $S p l \mathrm{I}=\mathrm{CGTACG}, E c o$ RI = GAATTC $, N d e \mathrm{I}=\mathrm{CATATG}, P s t \mathrm{I}=$ CTGCAG, BamHI = GGATCC. in Figure 3C. Compared with the wild-type strain Guy11, $\Delta$ moric8 mutants formed hypha melanin that was not as pigmented and possessed more aerial-hyphae.

To assess the ability of $\Delta$ moric8 mutants to cause rice blast disease, Q-3 and Q-10 were used to inoculate leaves of the susceptible barley cv. Golden Promise and rice cv. CO-39. The mutants were completely nonpathogenic on both susceptible barley and rice leaves consistent with the original observation of LY-130 (Fig. 4A through C), even when leaf surfaces were abraded (data not shown). These results demonstrated that the nonpathogenic phenotype of LY-130 mutant was caused by the T-DNA integration into the Guy11 genome and that MoRIC8 is essential for pathogenicity of Magnaporthe oryzae.

\section{MoRIC8 is required for infection-related morphogenesis.}

Deletion of MoRIC8 caused defects in vegetative growth, conidiation, appressorium formation, fertility, and penetration in M. oryzae (Table 3; Fig. 4). Deletion or disruption of MoRIC8 reduced fungal growth, conidiation, and appressorium formation but the emergence of germ tubes after conidial germination of LY-130 or Amoric8 mutants Q-3 and Q-10 was not affected (Table 3). Q-3, Q-10, and LY-130 mutants displayed reduced conidiation, producing $9.00 \pm 1.73,8.17 \pm 0.17$, and $11.00 \pm 0.33 \times 10^{4}$ conidia/plate, respectively, after a 10 -day incubation on CM (Talbot et al. 1993) at $25^{\circ} \mathrm{C}$ compared with Guy11 which produced $96.17 \pm 0.17 \times 10^{4}$ conidia/plate. Moreover, the formation of appressoria was significantly reduced in Q-3, Q-10, and LY-130 mutants compared with Guy11 (Table 3; Fig. 4D). Conidia of the wild-type strain Guy11 produced melanized appressoria after incubation on a hydrophobic surface, whereas most conidia of Q-3, Q-10, and LY-130 mutants formed long germ tubes that lacked germ tube tip differentiation (Fig. 4D). Even after prolonged incubation of up to $48 \mathrm{~h}$, tip differentiation and appressorium formation from germ tubes of Amoric8 and LY-130 mutants were not observed (data not shown). When conidia of the mutants were incubated on sterile onion epidermis they also formed long germ tubes, and very few appressoria were produced (Table 3; Fig. 4E). The long germ tubes produced by the mutants failed to penetrate onion epidermis (Fig. 4E), indicating that 4 moric8 and LY-130 mutants were affected in surface recognition, appressorium formation, and penetration on plant surfaces.

In order to determine the role of MoRIC8 in sexual reproduction, the wild-type Guy11 (MAT1-2), LY-130, and the $\Delta$ moric8 mutants Q-3 and Q-10 were crossed with a standard tester TH3 (MAT1-1) isolate of M. oryzae to allow perithecium production. After 3 weeks, the junctions between mated individuals were examined for the presence of perithecia. We observed numerous perithecia at the junctions of the wild-type strains of Guy11 and TH3 but no perithecia were produced from crossing Q-3 and TH3 or Q-10 and TH3 (Fig. 4F). Similarly, few perithecia were produced from crossing LY-130 and TH3 (Fig. 4F). We conclude that MoRIC8 is essential for fertility and sexual reproduction in $M$. oryzae.

When considered together, we conclude that MoRIC8 is required for multiple steps of plant infection-related morphogenesis, including vegetative growth, asexual sporulation, appressorium formation, and fertility in $M$. oryzae.

\section{MoRic8 localizes to the cytosol and is highly expressed during appressorium development.}

In order to investigate the temporal and spatial pattern of MoRIC8 expression during infection-related development, a $2.0-\mathrm{kb}$ promoter fragment upstream of the gene and the entire $M o R I C 8$ protein-coding sequence was fused in-frame to the green fluorescent protein (GFP)-encoding gene, GFP (sGFP), and introduced into LY-130 and a $\Delta$ moric8 mutant Q-10, respec- 


\section{A}

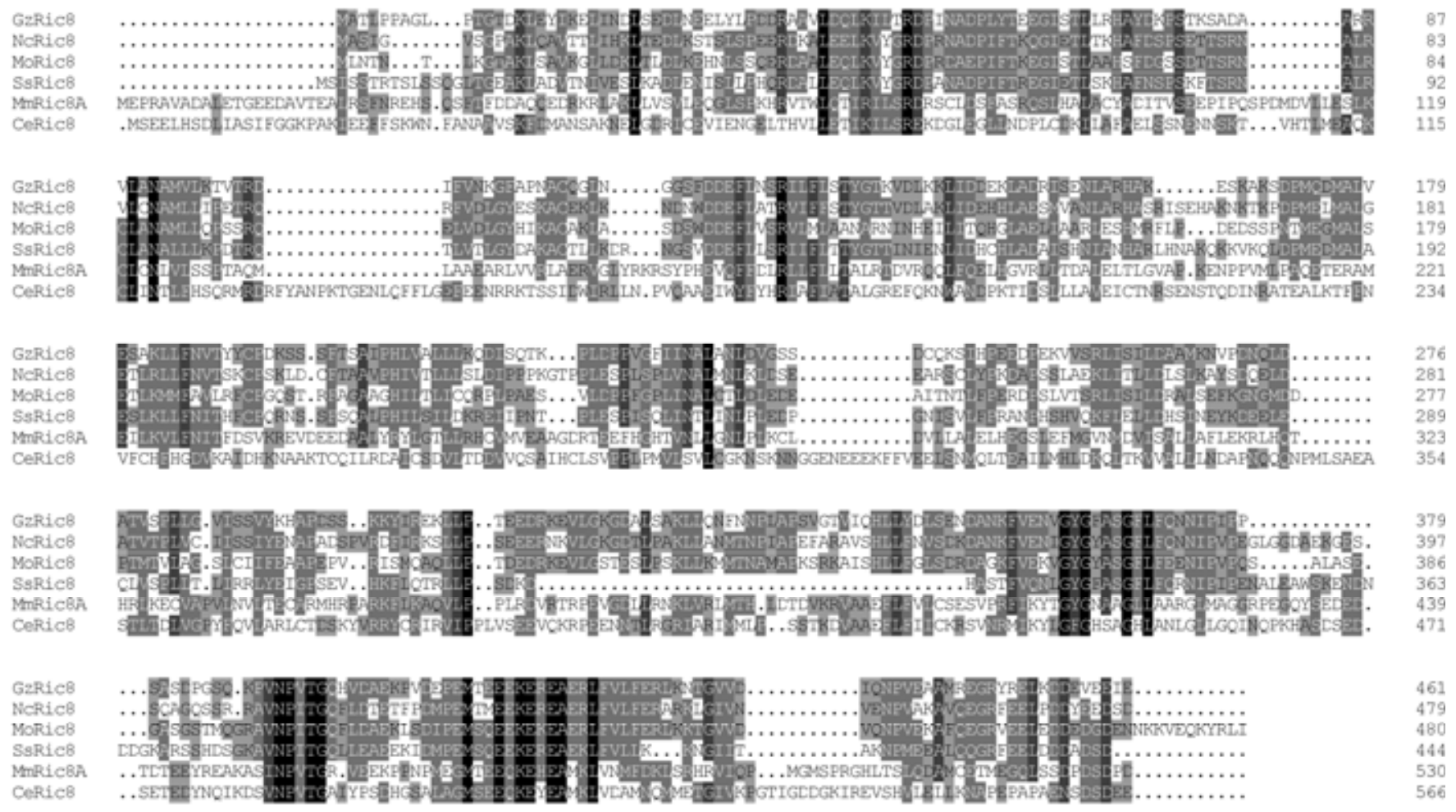

B

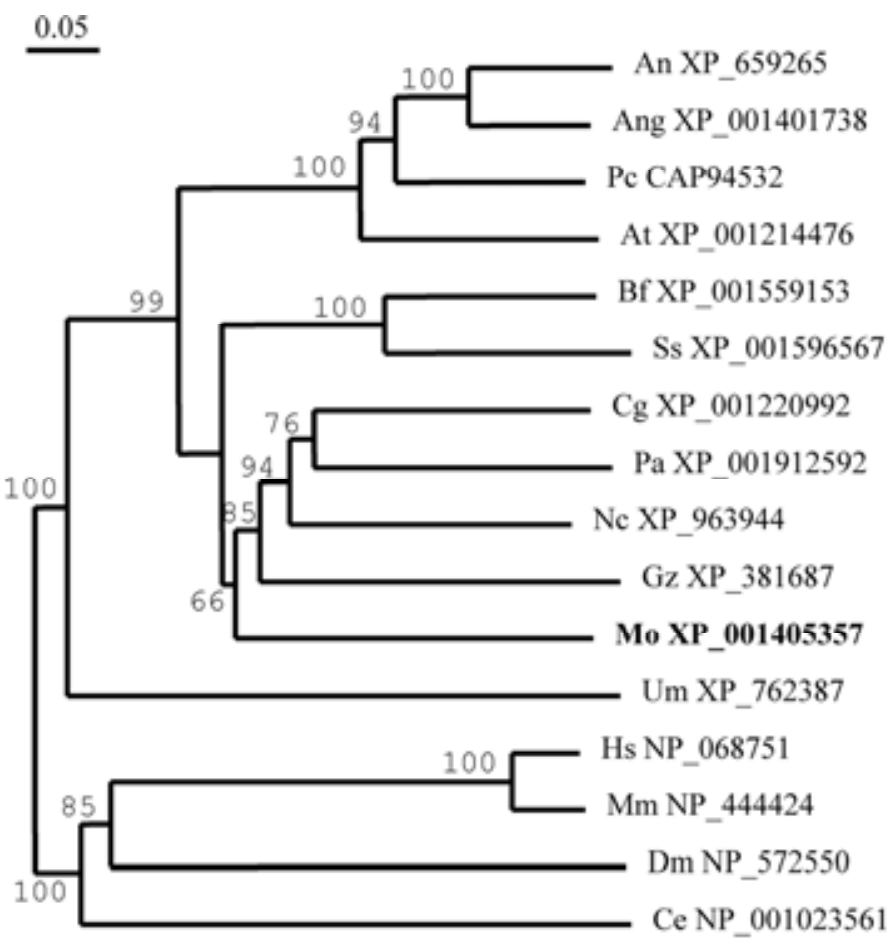

Fig. 2. Amino acid sequence alignment and phylogenetic analysis of Magnaporthe MoRic8 with the homologs from other species. A, Alignment of the predicted amino acid sequence of MoRic8 with its homologs from Neurospora crassa OR74A, Gibberella zeae PH-1, Sclerotinia sclerotiorum 1980, Caenorhabditis elegans, and Mus musculus (GenBank XP_963944, XP_381687, XP_001596567, NP_001023561, and NP_444424, respectively). Identical amino acids are shown on a black background and similar amino acids are shown on a light gray background. MoRic8, Magnaporthe oryzae MGG_14008; NcRic8, N. crassa predicted protein NCU02788 (Ric8); GzRic8, G. zeae predicted protein FG01511; SsRic8, S. sclerotiorum predicted protein SS1G_02787; CeRic8, C. elegans Ric8; MmRic8, Mus musculus Ric8A. B, Phylogenetic tree of Magnaporthe MoRic8 and 15 homologs from other species was constructed by observed divergency distance method in the program DNAMAN. Numbers at the nodes in the rooted tree represent bootstrapping value on 1,000 replications. Abbreviations and numbers correspond to species names and GenBank accession numbers, respectively. An $=$ Aspergillus nidulans; Ang $=$ A. niger; Pc $=$ Penicillium chrysogenum; $\mathrm{At}=$ A. terreus $; \mathrm{Bf}=$ Botryotinia fuckeliana $; \mathrm{Ss}=S$. sclerotiorum $; \mathrm{Cg}=$ Chaetomium globosum $; \mathrm{Pa}=$ Podospora anserine $; \mathrm{Nc}=$ N . crassa $; \mathrm{Gz}=$ G. zeae $; \mathrm{Mo}=$ Magnaporthe oryzae $; \mathrm{Um}=$ Ustilago maydis $; \mathrm{Hs}=$ Homo sapiens $; \mathrm{Mm}=$ Mus musculus $;$ Dm $=$ Drosophila melanogaster $;$ Ce $=$ Caenorhabditis elegans. The bar indicates 0.05 distance units. DNAMAN version5.2.2 program was used for alignment and phylogenetic tree construction. 
tively. Transformants carrying a single integration of the plasmid were selected by DNA gel-blot analysis (data not shown). An independent single RIC-GFP insertion event occurred in four transformants: LY-130-R1, LY-130-R4, Q-10C, and Q-10C3 (Table 1). Green fluorescence was observed in all these transformants. Q-10C was used to investigate gene expression patterns and the spatial localization of the MoRic8 protein. In this analysis, an evenly distributed faint GFP fluorescence was observed in the cytoplasm of mycelium and conidia (Fig. 5A), indicating that MoRic8-GFP fusion protein was expressed at a low level and localized in the cytosol.

To observe the MoRIC 8 expression patterns during appressorium development in $M$. oryzae, conidia of strain Q-10C was allowed to germinate on hydrophobic GelBond surfaces. GFP expression was observed by fluorescence microscopy at intervals of $4,8,16,24$, and 48 h. MoRIC $8-G F P$ was expressed during germ tube emergence and elongation. Once formed, appressoria of Q-10C were highly fluorescent (Fig. 5B). Green fluorescence was undetectable in the majority of conidia and germ tubes after incubation for $24 \mathrm{~h}$. However, even after $48 \mathrm{~h}$, $G F P$ was highly expressed in appressoria. Furthermore, MoRIC8-GFP expression patterns during appressorium development on sterile onion epidermis were similar to those on plastic surfaces (data not shown). Weak MoRIC8-GFP expression was present in some but not all invasive hyphae, indicating that MoRIC8 was expressed in infectious hyphae at a low level. Taken together, these data suggest that MoRic8 is cytoplasmic and abundantly expressed during appressorium development.

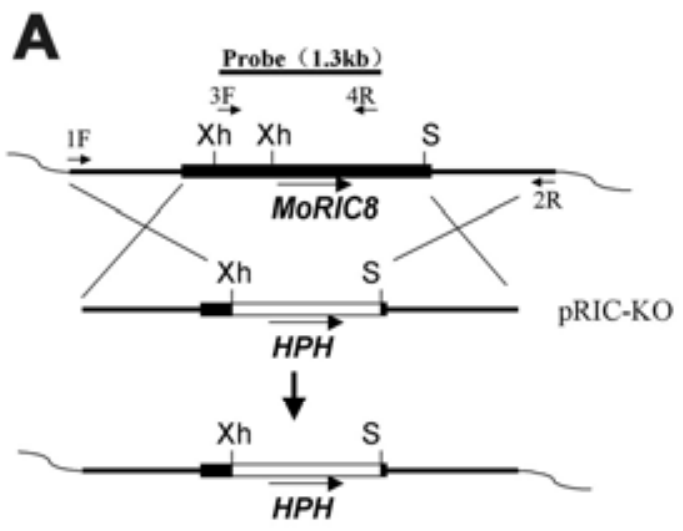

B
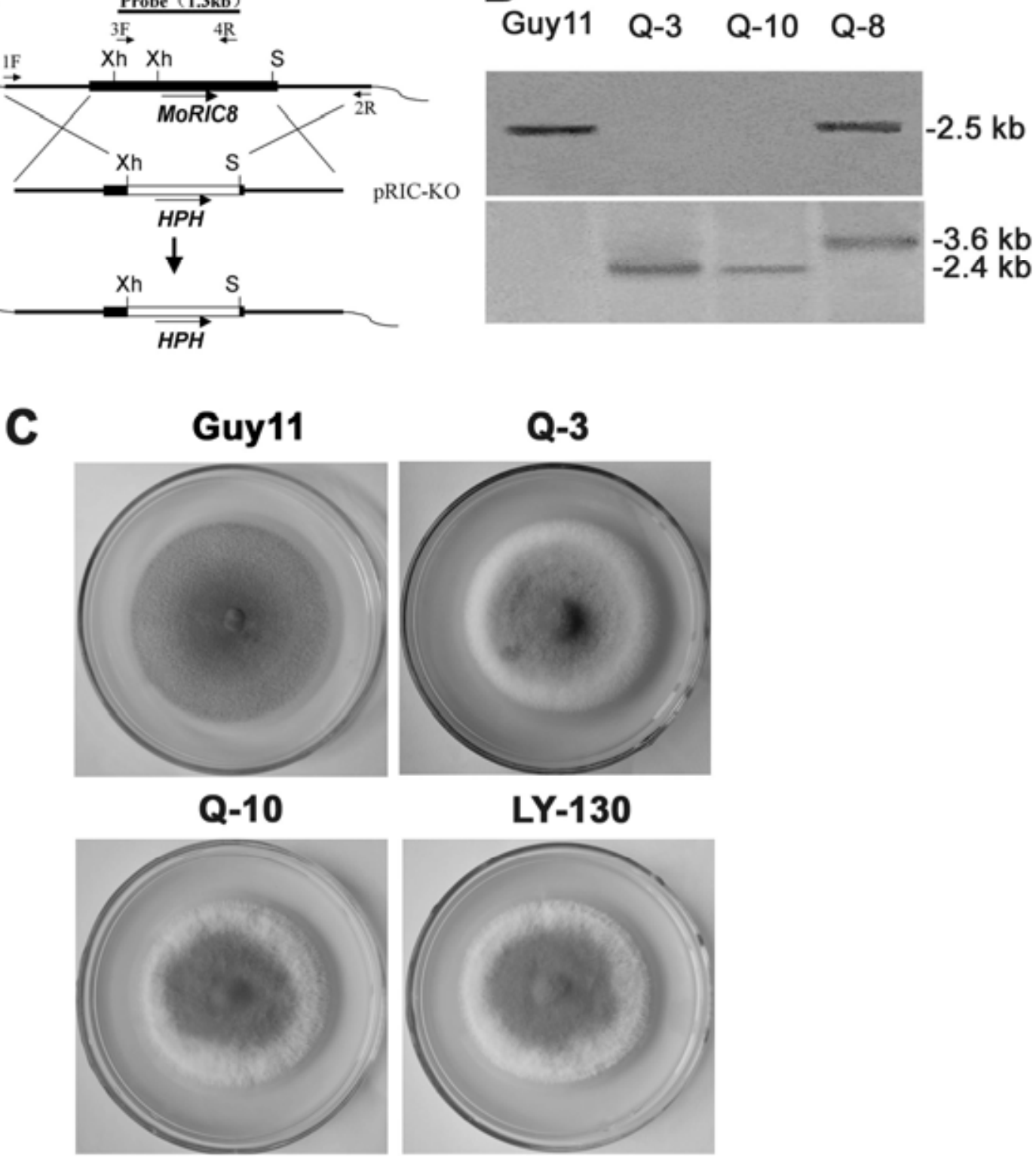

Fig. 3. Targeted gene replacement of MoRIC8. A, Construction of MoRIC8 gene replacement vector. A 3.6-kb fragment spanning the locus was amplified with the primers $1 \mathrm{~F}$ and $2 \mathrm{R}$ and subcloned to pGEM-T easy vector, and a 1.6-kb fragment digested with XhoI and SplI, which contained almost the entire MoRIC8 open reading frame, was removed. This was replaced with a 1.4-kb hygromycin B resistance gene cassette $(H P H)$ with the corresponding ends with $X h o I$ and SplI to generate gene replacement vector pRIC-KO. The resulting pRIC-KO was linearized and transformed into Magnaporthe oryzae Guyl1 for generating homologous recombinants. Xh $=$ XhoI; S = SplI. B, Amoric8 Mutants confirmed by Southern blot analysis. Genomic DNAs from the wild-type strain Guy11 and three transformants were digested with SalI and probed with a 1.3-kb fragment (top) amplified with the primers 3F and 4R and a 1.4-kb HPH cassette (bottom), respectively. Lane 1, Guy11; lanes 2 and 3, Amoric8 mutants Q-3 and Q-10; lane 4, ectopic transformant Q-8. C, Colonies of the wild-type strain Guy11, Amoric8 mutant Q-3, Q-10, and the T-DNA insertional mutant LY-130. Photographs were taken after incubating on CM (Talbot et al. 1993) at $25^{\circ} \mathrm{C}$ for 10 days. 
A

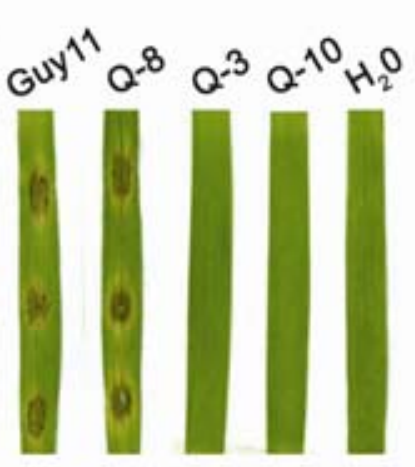

Barley

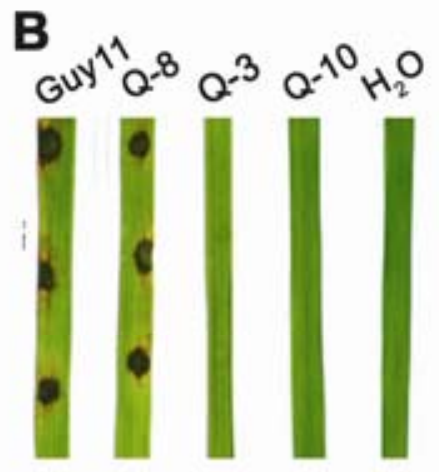

Rice

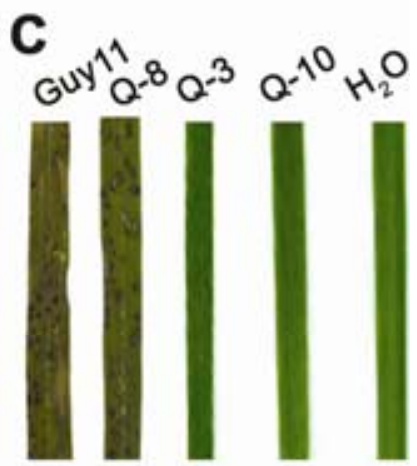

Rice
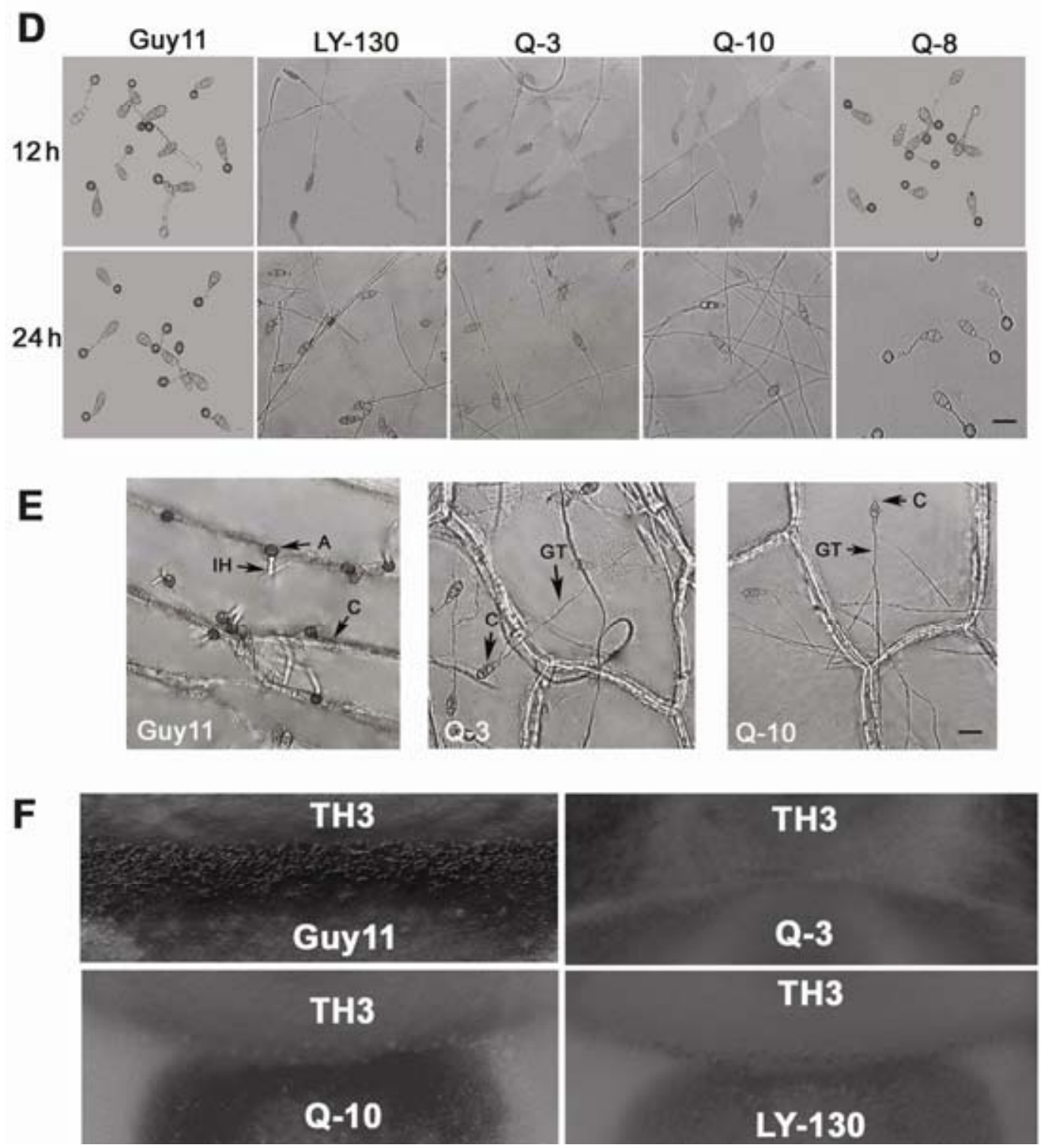

Fig. 4. Phenotypic analysis of $\Delta$ moric 8 mutants of Magnaporthe oryzae. A, B, and $\mathbf{C}, \Delta$ moric 8 mutants were completely nonpathogenic to susceptible barley and rice. A, Barley and $\mathbf{B}$, rice segments were inoculated with the conidial drops (10 $\mu \mathrm{l}$ per drop) from the wild-type strain Guy11, Q-3 ( $\Delta$ moric8), Q-10 ( $\triangle$ moric8), and Q-8 (ectopic transformant). $\mathrm{H}_{2} \mathrm{O}$ was used as the control. C, Rice leaves were spray inoculated with the conidia from the same strains. $\mathrm{H}_{2} \mathrm{O}$ containing $0.2 \%$ gelatin was used as the control. For all cases, conidia at a concentration $1 \times 10^{5}$ conidia ml $^{-1}$ were used and photographs were taken at 5 days after inoculation. No symptoms were observed on the leaves inoculated with $4 m o r i c 8$ mutants Q-3 and Q-10. D, $\Delta m o r i c 8$ Mutants Q-3 and Q-10 and TDNA integration mutant LY-130 were unable to form appressoria on GelBond films, whereas the wild-type strain Guy11 and ectopic transformant Q-8 formed numerous melanized appressoria. Bar $=10 \mu \mathrm{m}$. E, Amoric8 Mutants Q-3 and Q-10 forming long germ tubes without penetration occurred on onion epidermis at $24 \mathrm{~h}$ of incubation. Guy 11 developed normal appressoria and penetrated onion epidermal cells at $24 \mathrm{~h}$ of incubation. $\mathrm{C}=$ conidium; $\mathrm{A}=$ appressorium; IH = infectious hypha; GT = germ tube. Bar $=10 \mu \mathrm{m}$. F, Fertility assay. Guy $11 \times \mathrm{TH} 3$ forms numerous perithecia on oatmeal medium; no perithecia was observed for the crosses of Q- $3 \times \mathrm{TH} 3$ and Q-10 $\times$ TH3 and rare perithecia was produced for the cross of LY-130 $\times$ TH3. 


\section{Reintroduction of MoRIC8 into $\triangle$ moric8 mutants restores} the ability to cause rice blast disease.

To ensure that the phenotypic differences observed in LY130 or 4 moric 8 mutants were associated with the gene-replacement event, we carried out the phenotypic analysis of the complementation transformants LY-130-R1, LY-130-R4, Q-10C, and Q-10C3 (Table 1). All four of the GFP-expressing transformants exhibited full virulence to barley and rice by spray inoculation or using cut-leaf assays (data not shown). The phenotypes of LY-130 or Amoric8 mutants, including vegetative growth, conidiation, appressorium formation, fertility, and penetration, were fully complemented by reintroduction of the gene (Tables 3 and 4). We conclude that MoRIC8 is essential for multiple steps of plant infection-related morphogenesis development and pathogenicity in $M$. oryzae.

\section{MoRic8 acts upstream of the cAMP signaling pathway required for appressorium formation in M. oryzae.}

To test whether appressorium formation of LY-130, Q-3, and Q-10 mutants was responsive to exogenous chemicals, cAMP, 3-iso-butyl-1-methylxanthin (IBMX), and 1,16-hexadecanediol (Diol) were each added to the conidial suspensions. In the controls (without exogenous chemicals), the formation of appressoria was significantly reduced in Q-3 and Q-10 mutants compared with Guy11 (Fig. 6A through C). Interestingly, up to $60 \%$ of the germ tubes of LY-130, Q-3, or Q-10 were induced to form appressoria on GelBond films when supplemented with cAMP (Table 4; Fig. 6D and E). However, the appressoria induced by adding exogenous cAMP were unable to penetrate onion epidermal cells and did not cause lesions on rice leaves (Fig. 6K through M). In contrast, Guy11 appressoria formed upon addition of cAMP, penetrated onion epidermal cells, and developed infectious hyphae (Fig. 6J) with similar patterns to the treatment without adding cAMP (Fig. 4E), and numerous typical lesions were produced on spray-inoculated rice leaves (Fig. 6M). IBMX is an inhibitor of phosphodiesterase activity and, when IBMX was used as an inducer for appressorium formation of $\Delta$ moric 8 mutants, more than $79 \%$ of the germ tubes of LY-130, Q-3, or Q-10 were induced to form appressoria by $24 \mathrm{~h}$ on hydrophobic GelBond (Table 4; Fig. 6F and G). Similarly, the induced appressoria of $\Delta$ moric 8 mutants by adding IBMX were nonpathogenic on rice (data not shown). However, adding $1 \mu \mathrm{M}$ Diol to conidial suspensions did not induce the appressorium formation in LY-130 and Amoric8 mutants (Table 4; Fig. $6 \mathrm{H}$ and I). These results indicate that MoRIC8 is involved in cAMP signaling during appressorium formation in M. oryzae.

\section{MoRic8 physically interacts}

with the MagB G $\alpha$ subunit protein.

In view of the effect of cAMP on the observed mutant phenotypes, we reasoned that MoRic8 might be involved in signal transduction prior to activation of adenylate cyclase. To test this idea, we investigated the genes coding $\mathrm{G} \alpha, \mathrm{G} \beta$, and $\mathrm{G} \gamma$ subunits by quantitative (q)RT-PCR. Expression of the three genes ( $M A G A, M A G B$, and $M A G C$ ) encoding $\mathrm{G} \alpha$ subunits was significantly $(P<0.01)$ downregulated in the $\Delta$ moric 8 mutants Q-3 and Q-10 (Fig. 7). Expression of $M G G 1$ encoding G $\gamma$ subunit was also significantly $(P<0.05)$ downregulated in the mutants (Fig. 7). However, expression of $M G B l$ encoding the $\mathrm{G} \beta$ subunit was not significantly affected in the mutants (Fig. 7). These results suggest that deletion of MoRIC8 leads to lower expression of the three $\mathrm{G} \alpha$ subunit genes and the $\mathrm{G} \gamma$ subunit gene but does not significantly affect expression of the gene encoding the $\mathrm{G} \beta$ subunit.

To test whether MoRic8 physically interacts with the $\mathrm{G} \alpha$ proteins (MagA, MagB, or MagC), we carried out Y2H experiments. The full-length MoRIC8 was cloned into the prey vector and the G $\alpha$ protein genes were cloned into the bait vectors, respectively. The resulting prey and each bait vector were cotransformed into yeast strain AH109. Yeast transformants were then assayed for growth on SD-Trp-Leu-His-Ade medium at specified concentrations. In the $\mathrm{Y} 2 \mathrm{H}$ assays, MoRic8 directly interacts with MagB but not MagA and MagC (Fig. 8). In addition, no physical interactions was observed between MoRic8 and Mgb1 or Mgg1 in Y2H assays (data not shown). These results indicated that MoRic8 directly interacts with $\mathrm{G} \alpha$ subunit protein MagB but not with the other G-proteins.

\section{DISCUSSION}

Insertional mutagenesis is an effective way to identify novel genes involved in plant infection-related morphogenesis and pathogenicity in fungal pathogens, including M. oryzae (Wilson and Talbot 2009). In this report, we identified novel virulent determinants of $M$. oryzae using T-DNA insertional mutagenesis. LY-130 was identified as a nonpathogenic mutant on barley and rice. HiTAIL PCR revealed that the integrated T-DNA in the mutant disrupted a genomic region corresponding to a locus putatively encoding protein with similarity to Ric8/Ric8A (named for resistance to inhibitors of cholinesterase/synembryn), previously described in C. elegans, Mus musculus, and Drosophila melanogaster (Miller et al. 1996, 2000; Tall et al. 2003; Afshar et al. 2004; Siderovski and Willard 2005; Wang et al. 2005). Ric8/Ric8A acts as a G-protein-coupled receptor (GPCR)-independent guanine nucleotide exchange factor (GEF) for some $\mathrm{G} \alpha$ subunits and plays a significant role in asymmetrical cell division and synaptic vesicle priming (Afshar et al. 2004; Siderovski and Willard 2005). The RIC8 gene is highly conserved evolutionarily but the encoded protein has no obvious domain or motif structure (Siderovski and Willard 2005). M. musculus Ric8A (GenBank: NP_444424) showed 87\% amino acid identity with Homo sapiens Ric8 (GenBank:

Table 3. Phenotypic analysis of $\Delta$ moric8 mutants of Magnaporthe oryzae

\begin{tabular}{|c|c|c|c|c|c|c|c|}
\hline \multirow[b]{2}{*}{ Strain } & \multirow[b]{2}{*}{ Colony $(\mathrm{cm})^{d}$} & \multirow[b]{2}{*}{ Conidiation $\left(\times 10^{4}\right)^{\mathrm{e}}$} & \multicolumn{2}{|c|}{ Germination $(\%)^{b}$} & \multicolumn{2}{|c|}{ Appressorium (\%) } & \multirow[b]{2}{*}{ Penetration $(\%)^{\mathrm{f}}$} \\
\hline & & & $2 \mathrm{~h}$ & $4 \mathrm{~h}$ & GB & $\mathbf{O E}$ & \\
\hline Guy11 & $6.95 \pm 0.01$ & $96.17 \pm 0.17$ & $62.23 \pm 0.03$ & $97.11 \pm 0.02$ & $96.41 \pm 0.57$ & $95.77 \pm 0.24$ & $83.78 \pm 0.02$ \\
\hline LY-130 & $5.93 \pm 0.07$ & $11.00 \pm 0.33$ & $56.78 \pm 0.06$ & $96.25 \pm 2.62$ & $15.92 \pm 1.55$ & $14.03 \pm 0.29$ & 0 \\
\hline Q-3 & $6.01 \pm 0.10$ & $9.00 \pm 1.73$ & $60.39 \pm 1.49$ & $95.94 \pm 0.95$ & $4.93 \pm 1.29$ & $4.49 \pm 0.76$ & 0 \\
\hline Q-10 & $5.81 \pm 0.06$ & $8.17 \pm 0.17$ & $62.69 \pm 0.04$ & $96.44 \pm 0.02$ & $3.17 \pm 0.51$ & $3.67 \pm 0.13$ & 0 \\
\hline Q-10C & $6.98 \pm 0.06$ & $94.50 \pm 0.17$ & $65.53 \pm 1.34$ & $96.89 \pm 1.08$ & $95.86 \pm 0.17$ & $95.64 \pm 0.48$ & $81.67 \pm 3.51$ \\
\hline
\end{tabular}

${ }^{a}$ Data shown are the mean values obtained from three independent experiments and the standard deviation is given.

${ }^{\mathrm{b}}$ Percentage of conidia produced germ tubes at time intervals of 2 and $4 \mathrm{~h}$ on GelBond surface.

${ }^{c}$ Percentage of appressorium formation on GelBond film (GB) or onion epidermis (OE) surfaces after incubation for $24 \mathrm{~h}$ at $25^{\circ} \mathrm{C}$.

${ }^{\mathrm{d}}$ Diameter of colonies grown on CM plates (Talbot et al. 1993) at $25^{\circ} \mathrm{C}$ for 10 days.

e Number of conidia per plate from 10 -day-old CM cultures at $25^{\circ} \mathrm{C}$.

${ }^{\mathrm{f}}$ Percentage of penetration hypha formation from appressoria after incubation on onion epidermis for $24 \mathrm{~h}$ at $25^{\circ} \mathrm{C}$. More than 300 spores or appressoria were counted for each replicate per strain. 
NP_068751). However, C. elegans Ric8 (GenBank: NP 001023561) showed only 24.2 and $23.5 \%$ amino acid identity with $M$. musculus Ric8A and $H$. sapiens Ric8, respectively. Magnaporthe oryzae MoRic8 showed a low amino acid identity with Mus musculus Ric8A and C. elegans Ric8 (Fig. 2A), indicating the evolutionary distance of the RIC8 gene between fungi and animals. Consistently, the genetic relationship between MoRic8 and Ric8/Ric8A from animal species is, indeed, relatively distant (Fig. 2B). However, the relationship between MoRic8 and Ric8/Ric8A homologs from other fungi, including those in $N$. crassa and G. zeae, is, as expected, much closer (Fig. 2A and B), with 49.0 and $43.3 \%$ amino acid identity, respectively. Interestingly, although Ric8 is present in filamentous fungi, it is absent from the genomes of $S$. cerevisiae, protists, or plants. To our knowledge, Ric8/Ric8A homologs have not been functionally characterized in phytopathogenic fungi.

Our results showed that MoRIC 8 is required for the rice blast disease and multiple infection-related developmental processes in Magnaporthe oryzae, such as vegetative growth, conidiation, appressorium formation, and fertility. Nonpathogenic phenotypes of LY-130 or Amoric8 mutants may be partially due to the significant reduction in appressoria produced and by loss of appressorial function. However, based on the fact that the mutants failed to form lesions even on wounded leaves, MoRIC8 also appears to contribute to invasive growth in host cells. Plant cutin monomers such as Diol are known to promote appressorium formation in $M$. oryzae. However, adding external Diol to conidial suspensions of LY-130 or Amoric8 mutants did not restore the defect in appressorium formation on hydrophobic surfaces. In previous reports, appressorium formation of $\mathrm{magB}$ mutants was restored by addition of external Diol (Liu and Dean 1997). Thus, the roles of Diol on triggering appressorium formation appear to be dependent on MoRic8 but not MagB.

Adding exogenous cAMP or IBMX to conidial suspensions of LY-130 or Amoric8 mutants did induce appressorium development, consistent with MoRic8 acting upstream of cAMP generation (Fig. 6D, E, K, and L; Table 4). However, the appressoria induced were unable to penetrate cuticles and to cause blast disease (Fig. 6M). This is consistent with results of disruption mutants of $M A G B$. For example, for $\triangle m a g B$ mutants, less than $5 \%$ of the conidia could be induced to form appressoria on hydrophobic surface and this defect of the mutants could be rescued by adding exogenous cAMP or IBMX (Liu and Dean 1997). The induced appressoria were also nonpathogenic. Obviously, the defects of appressorium formation and pathogenicity due to the deletion of MoRIC8 were very similar to those of the disruption mutants of MAGB. Deletion of MoRIC 8 also blocked appressorium formation as well as sexual development. The $\Delta$ moric 8 mutants prevented initiation of mating processes and failed to form perithecia when crossed with the appropriate matingtype strain. This suggests that MoRIC 8 is involved in the sexual pathway induced by sex pheromone effectors. Similarly, the $\triangle m a g B$ mutants were also unable to produce perithecia (Liu and Dean 1997). These results implicate MoRIC8 in activation of the cAMP-dependent signaling pathway mediated by G-proteins for surface recognition, appressorium formation, and fertility in $M$. oryzae.

In eukaryotic cells, heterotrimeric G-protein signaling is essential for sensing environmental changes. The inactive heterotrimer consists of $\mathrm{G} \alpha, \mathrm{G} \beta$, and $\mathrm{G} \gamma$ subunits in association with a GPCR, which acts as a GEF. Recognition of a ligand with the GPCR causes exchange of GTP for GDP and activation of the G $\alpha-G T P$ and G $\beta \gamma$ (Neves et al. 2002; Li et al. 2007). Ric8, a GPCR-independent GEF for G $\alpha$, was originally found by genetic studies of C. elegans mutants (Miller et al. 1996, 2000).
Afterward, two homologs of the C. elegans protein Ric8, Ric8A and Ric8B, were isolated in a Y2H screen for $\mathrm{G \alpha}_{\mathrm{s}^{-}}$and $\mathrm{G \alpha}_{0^{-}}$ interacting proteins in rat (Tall et al. 2003). In mammalian cells, Ric8/Ric8A has been demonstrated to be associated with some $\mathrm{G} \alpha$ proteins (Tall et al. 2003). When a G $\alpha$ protein is bound to GDP, binding of Ric8/Ric8A causes rapid release of GDP and forms a stable nucleotide-free Ric8/Ric8A:G $\alpha$ complex. Unlike a GPCR, Ric8/Ric8A does not contain transmembrane spanning sequences. In this report, our results have shown that MoRIC8 is involved in the cAMP-dependent signaling pathway and is required for vegetative growth, conidiation, appressorium formation, fertility, and pathogenicity in $M$. oryzae.

To our knowledge, Ric8 orthologues of phytopathogenic fungi have not been well characterized. A requirement for Ric8 in vegetative growth, conidiation, and sexual development has been observed very recently in $N$. crassa (Schacht and Borkovich 2009; Wright et al. 2009). Deletion of RIC8 in $N$. crassa leads to defects similar to phenotypes observed for lacking the G $\alpha$ genes GNA-1 and GNA-3 (Wright et al. 2009), which are homologous with $M A G B$ and $M A G A$, respectively. In addition, $N$. crassa Ric8 interacts with Gna-1 and Gna-3 in a Y2H assay (Wright et al. 2009). However, deletion of MAGA in $M$. oryzae had no effect on vegetative growth, conidiation, or appressorium formation, although it blocked production of mature asci (Liu and Dean 1997). In this report, we found that MoRic8 directly interacts with the G $\alpha$ subunit MagB using the Y2H assay. However, no physical interactions were detected between MoRic8 and the other G-proteins (MagA, MagC, Mgb1, and Mgg1).

When considered together, our results suggest that MoRic8 acts within the cAMP-response pathway by interaction with MagB and, therefore, may be a key component of the regulatory pathway that allows $M$. oryzae to form appressorium on hydrophobic surfaces.

More experimental evidence will be required for further insight into the role of MoRic8 in G-protein signaling pathways. A recent report has demonstrated that AC5 (type V adenylate cyclase) interacts with Ric8A through directly interacting at its $\mathrm{N}$-terminus, and the activity of AC5 via a $\mathrm{G \alpha}_{\mathrm{i}}$-dependent pathway is suppressed by the binding of Ric8A to the $\mathrm{N}$ terminus of AC5 (S. C. Wang et al. 2007, 2009). Although it is not entirely clear that release of free $G \beta \gamma$ subunits is a mechanism that occurs within these Ric8/G-protein signaling pathways, it is worth speculating that a potential function of Ric8 is to enhance G $\beta \gamma$ subunit signaling (Malik et al. 2005). Thus, the interaction between MoRic8 and Mac1 will need to be clarified, and the roles of MoRic8 in the M. oryzae Pmk1 MAPK pathway in regulating appressorium development will also be investigated in future.

Table 4. Effects of different inductive cues on appressorium formation of $\Delta$ moric8 mutants

\begin{tabular}{lrcc}
\hline & \multicolumn{3}{c}{ Chemical inducers $^{\mathbf{a}}$} \\
\cline { 2 - 4 } Strain & \multicolumn{1}{c}{ Diol } & cAMP & IBMX \\
\hline Guy11 & $95.76 \pm 0.15$ & $95.81 \pm 0.68$ & $96.05 \pm 0.60$ \\
LY-130 & $13.54 \pm 0.58$ & $60.77 \pm 2.46$ & $80.22 \pm 0.39$ \\
Q-3 & $4.07 \pm 1.02$ & $61.11 \pm 2.33$ & $79.17 \pm 2.97$ \\
Q-10 & $3.83 \pm 0.13$ & $60.41 \pm 1.83$ & $81.07 \pm 0.41$ \\
Q-10C & $95.79 \pm 0.18$ & $96.29 \pm 0.27$ & $95.67 \pm 0.88$ \\
\hline
\end{tabular}

${ }^{a}$ Conidia were incubated at $5 \times 10^{4}$ spores $\mathrm{ml}^{-1}$ for $24 \mathrm{~h}$ on hydrophobic GelBond films and inducers of the cutin monomer 1,16-hexadecanediol (Diol), cyclic adenosine 3',5'-cyclophosphate (cAMP), and 3iso-butyl-1-methylxanthin (IBMX) were added to final concentrations of $1 \mu \mathrm{M}, 10 \mathrm{mM}$, and $2.5 \mathrm{mM}$, respectively. Data shown are the mean values obtained by three independent experiments, and the standard deviation is given. More than 300 spores were counted for each replicate per strain. 
A

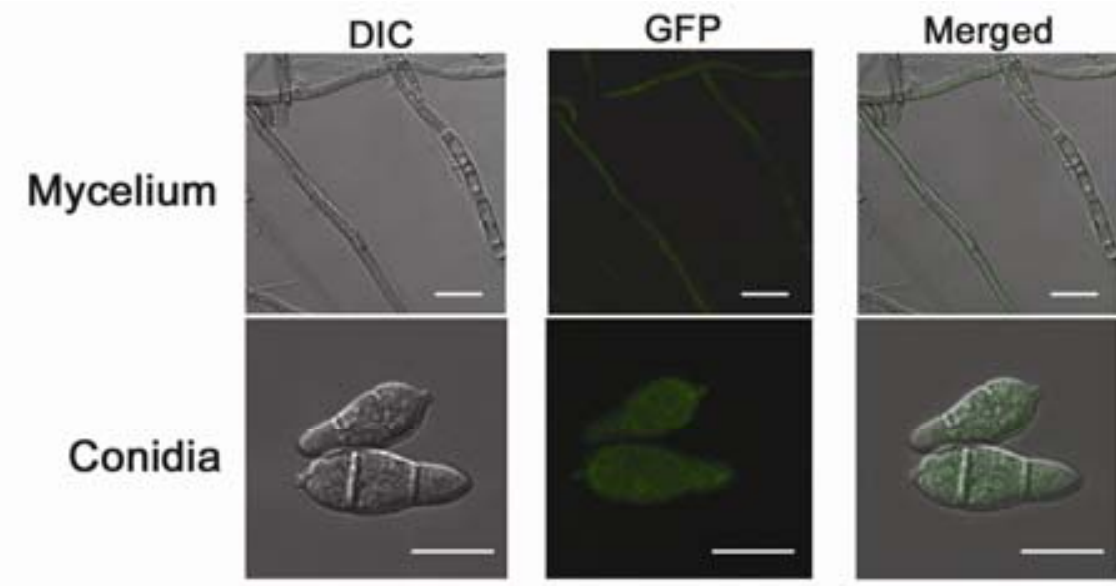

B

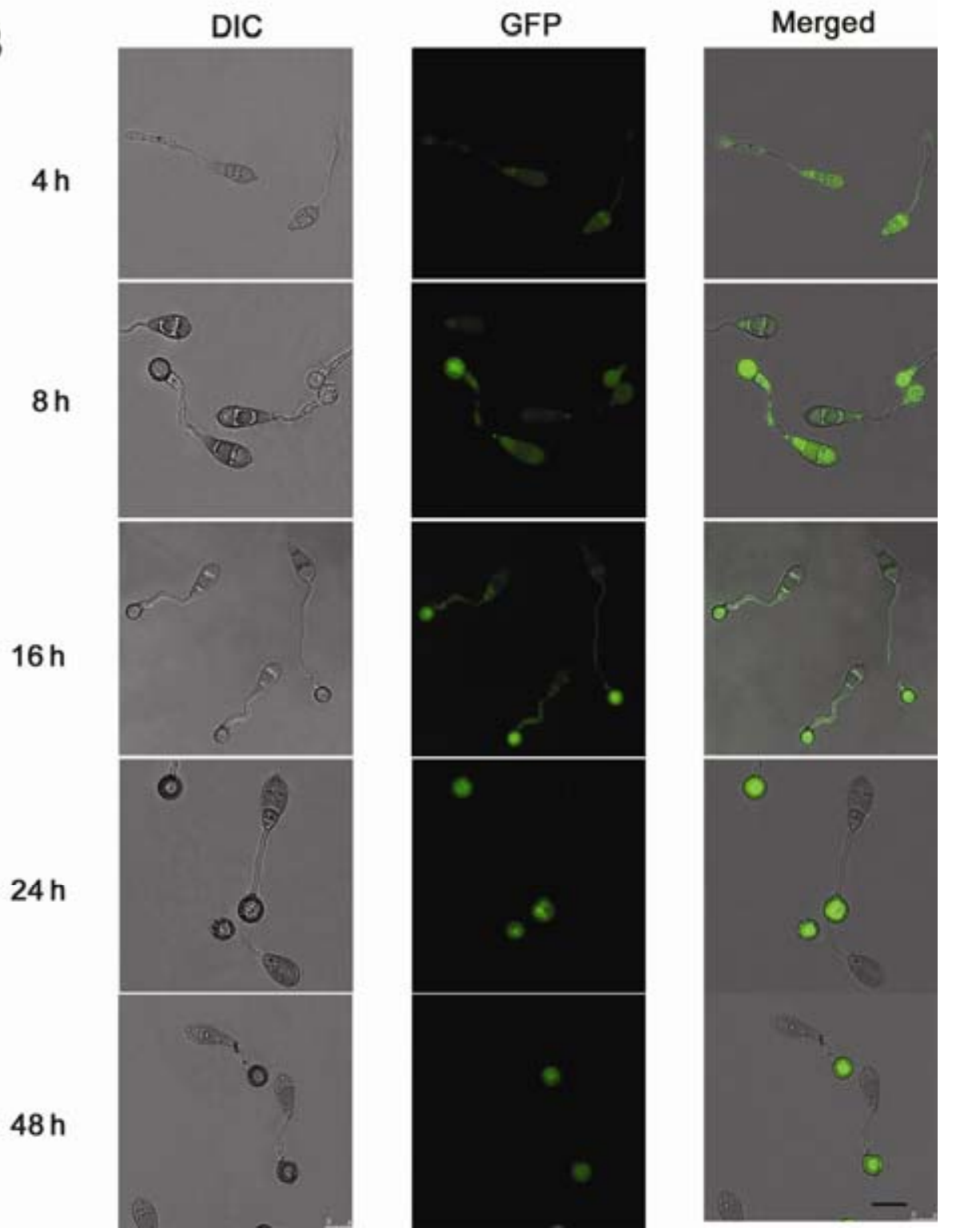

Fig. 5. Temporal and spatial expression of Magnaporthe oryzae MoRIC8. A, Expression of M. oryzae MoRIC8 in mycelia and conidia of Q-10C. MoRic8 Cterminal green fluorescent protein (GFP) fusion strategy was conducted to generate GFP expression transformants (see Material and Methods). Transformants LY-130-R1, LY-130-R4, Q-10C, and Q-10C3 carrying single-copy integrations of the MoRIC8::GFP::ILV1 transgene were identified. Q-10C was used for the expression analysis of M. oryzae MoRIC8 in mycelia and conidia by a confocal laser scanning microscope. DIC = differential interference contrast. Mycelia and conidia were harvested from a 10-day-old culture on CM (Talbot et al. 1993) plate. An evenly distributed GFP fluorescence in the cytoplasm of mycelium and conidia was observed. Bar $=10 \mu \mathrm{m}$. B, Expression of M. oryzae MoRIC8 during conidial germination and appressorium development. Q-10C was used for expression of M. oryzae MoRIC8-GFP during conidial germination and appressorium development. Conidia were allowed to germinate on hydrophobic GelBond film surfaces. Photographs were taken at intervals $(4,8,16,24$, and $48 \mathrm{~h})$. Bar $=10 \mu \mathrm{m}$. 

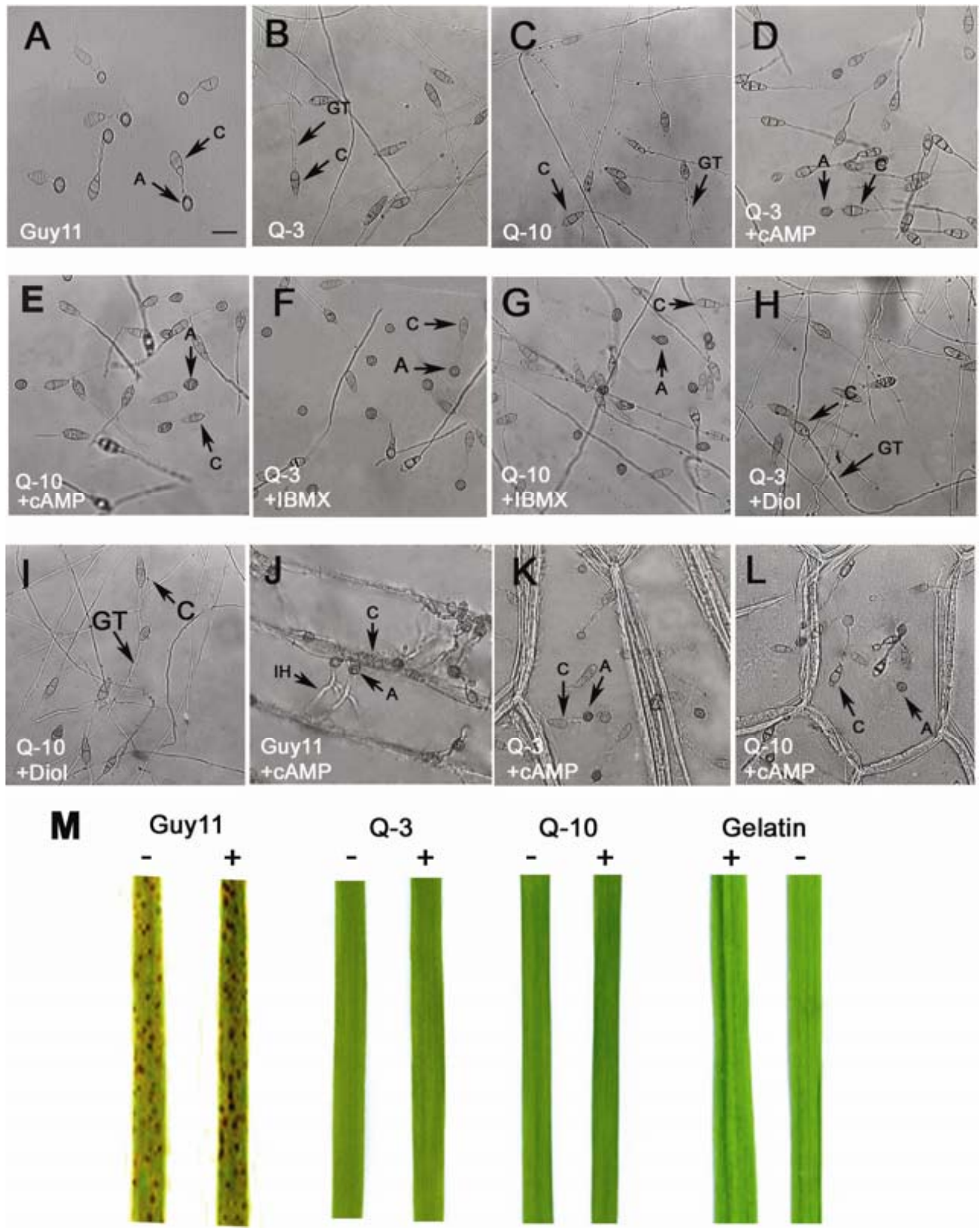

Fig. 6. Appressorium formation of $\Delta$ moric 8 mutants induced by adding exogenous cyclic adenosine $3^{\prime}, 5^{\prime}$-cyclophosphate (cAMP) and 3-iso-butyl-1-methylxanthin (IBMX). Conidia were allowed to germinate on hydrophobic side of $\mathbf{A}$ through $\mathbf{I}$, GelBond films or $\mathbf{J}$ through $\mathbf{L}$, onion epidermis in the presence or absence of different inducers at $25^{\circ} \mathrm{C}$. Photographs were taken at $24 \mathrm{~h}$ after incubation. $\mathrm{C}=$ conidium; GT $=$ germ tube; $\mathrm{A}=$ appressorium. Bar $=10 \mu \mathrm{m}$. A, Guy11 formed melanized appressoria. B and C, $\Delta$ moric8 Mutants Q-3 and Q-10 formed long germ tubes. D and E, Adding $10 \mathrm{mM}$ exogenous cAMP into spore suspensions of Q-3 and Q-10 partially remediated appressorium development. F and G, Adding $2.5 \mathrm{mM}$ IBMX into spore suspensions of Q-3 and Q10 partially remediated appressorium development. $\mathbf{H}$ and I, Adding $1.0 \mu \mathrm{M}$ 1,16-hexadecanediol (Diol) into spore suspensions of Q-3 and Q-10 formed long germ tubes. $\mathbf{J}$, Appressorium formation and penetration on onion epidermis by adding $10 \mathrm{mM}$ cAMP into spore suspension of Guy 11 . K and $\mathbf{L}$, Adding $10 \mathrm{mM}$ exogenous cAMP into spore suspensions of Q-3 and of Q-10 partially remediated appressorium development on onion epidermis but no penetration was observed. M, Pathogenicity tests of $\triangle$ moric 8 mutants by adding exogenous cAMP. Rice seedlings were spray inoculated with conidia from the wildstrain Guy11, Q-3, or Q-10 with (+) or without (-) 10 mM cAMP; $0.2 \%$ gelatin was used as the negative control. Typical disease symptoms only appeared on leaves inoculated with Guy11. Photographs were taken at 5 days after inoculation. 


\section{MATERIALS AND METHODS}

Strains, culture conditions, and DNA analysis.

All mutants described in the present study were generated from the M. oryzae wild-type strain Guy11 (Table 1). Standard growth and storage procedures for fungal strains were performed, as described previously (Talbot et al. 1993). Escherichia coli DH-5 $\alpha$ was used for routine bacterial transformations and maintenance of various plasmids in this study. A. tumefaciens AGL1 was used for T-DNA insertional transformation. Southern blot analysis was performed by the digoxigenin (DIG) high prime DNA labeling and detection starter Kit I (Roche, Mannheim, Germany). General procedures for nucleic acid analysis followed standard protocols (Sambrook et al. 1989).

\section{ATMT.}

Transformation of conidia and selection of hygromycinresistant transformants of $M$. oryzae were performed using ATMT (de Groot et al. 1998; Rho et al. 2001). A binary vector for T-DNA insertional mutagenesis was constructed following a procedure similar to the construction of $\mathrm{pBHt} 2$ described previously (Mullins et al. 2001). The hygromycin-resistance gene cassette (Carroll et al. 1994) was obtained from pCB1003 with $H p a \mathrm{I}$ digestion and then integrated into $\mathrm{XhoI}$ and $B s t \mathrm{XI}$ sites of pCAMBIA1300 to generate the binary vector. A. tumefaciens AGL1 containing the binary vector was grown at $28^{\circ} \mathrm{C}$ overnight in $5 \mathrm{ml}$ of Luria-Bertani medium containing kanamycin at $50 \mu \mathrm{g} \mathrm{ml}^{-1}$. The bacterial cells were harvested and diluted to an optical density at $600 \mathrm{~nm}=0.15$ in induction medium (Bundock et al. 1995) and then grown for $6 \mathrm{~h}$. Conidia harvested from 10-day-old cultures were adjusted to $1 \times 10^{6}$ spores $\mathrm{ml}^{-1}$ and co-cultivated in the presence of $200 \mu \mathrm{M}$ acetosyringone with A. tumefaciens cells. Selection of hygromycinresistant transformants of $M$. oryzae was made using $\mathrm{CM}$ medium containing hygromycin at $250 \mu \mathrm{g} \mathrm{m}{ }^{-1}$ (Talbot et al. 1993). The transformants were further purified by single-spore isolation and confirmed on CM plates containing hygromycin at $150 \mu \mathrm{g} \mathrm{ml}^{-1}$.

\section{Construction of vectors and fungal transformation.}

For construction of the MoRIC 8 gene replacement vector, a $3.65-\mathrm{kb}$ fragment spanning the MoRIC8 locus was amplified with primers $1 \mathrm{~F}$ and $2 \mathrm{R}$ (Table 2; Fig. 3A) and cloned into pGEM-T easy vector (Promega, Madison, WI, U.S.A.), and a $1.57-\mathrm{kb} X h o \mathrm{I}$ and SplI fragment containing the majority of the MoRIC8 ORF was removed and replaced sequentially with the 1.4-kb $H P H$ gene cassette, which encodes hygromycin phosphotransferase under control of the Aspergillus nidulans TrpC promoter (Carroll et al. 1994), amplified with primers HPH$\mathrm{Xh}-\mathrm{F}$ and HPH-Sp-R (Table 2) using pCB1003 as a template. The resulting plasmid pRIC-KO was digested with NotI to liberate a linear gene disruption cassette, which was used to transform protoplasts of $M$. oryzae Guy11 protoplasts, as described previously (Talbot et al. 1993).

The C-terminal GFP-tagging vector, pRIC-GFP, was constructed by amplification of 3.9-kb fragment, including a 1.9$\mathrm{kb}$ MoRIC8 gene-coding sequence and a $2.0-\mathrm{kb}$ promoter region, using primers HB-F and HB-R (Table 2). Construction of pRIC-GFP was carried out as follows. The 3.9-kb PCR product was cloned into pGEM-T easy vector to generate pGMRIC. The 1.5-kb GFP allele (Chiu et al. 1996) carrying the $A$. nidulans trpC terminator was amplified using primers GFP-F

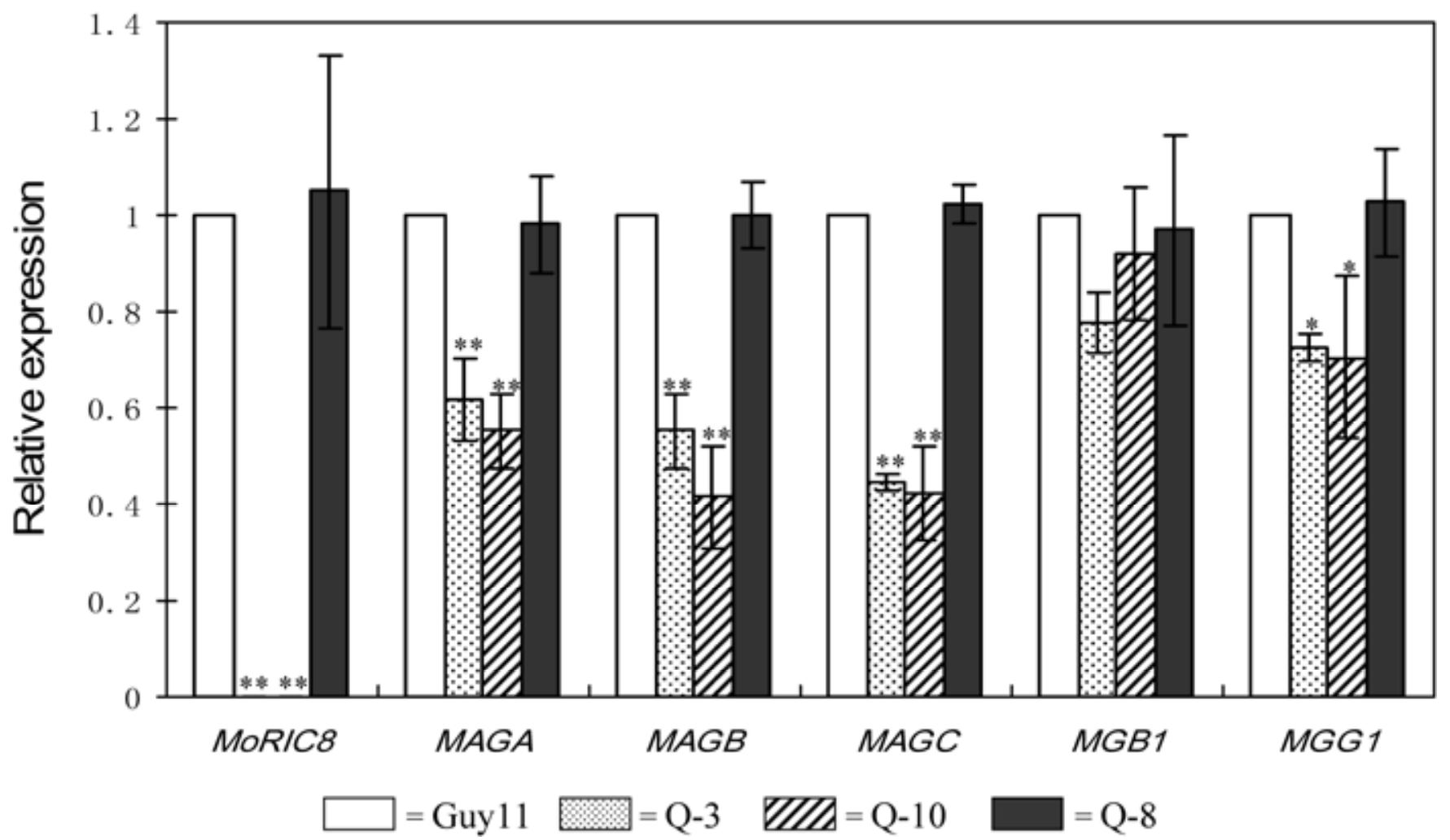

Fig. 7. Expression of the genes coding GTP-binding protein (G-protein) subunits in the Amoric 8 mutants measured by quantitative reverse-transcription polymerase chain reaction (qRT-PCR). Expression of the genes coding G-protein subunits in the 4 moric8 mutants Q-3, Q-10, and ectopic transformant Q-8 was measured using qRT-PCR. The abundance of the gene transcripts was calculated relative to endogenous control ( $\beta$-tubulin gene) using the $2^{-\Delta \Delta C T}$ method (Livak and Schmittgen 2001). The level of gene expression in Guy11 was taken as 1 and the relative expression in the mutants Q-3, Q-10, and ectopic transformant Q-8 was normalized based on 1. Error bars represent the standard deviation. Asterisks (* and ** $=P<0.05$ and 0.01 , respectively) indicate a significant difference of gene expression between Guy11 and Q-3, Q-10, or Q-8. Expression of the three G $\alpha$ subunit genes $(M A G A, M A G B$, and MAGC) and the $\mathrm{G} \gamma$ subunit gene (MGG1) was significantly affected by the deletion of MoRIC8. 
and GFP-R (Table 2), cloned to pGEM-T easy vector, and then digested with $K p n I$ to release the GFP allele with $K p n I$ ends, which was used to insert the KpnI site of pGM-RIC to create pGM-RIC-GFP. The pGM-RIC-GFP vector was checked by DNA sequencing to confirm the correct orientation of GFP insertion and in-frame fusion. Finally, pGM-RIC-GFP was digested with NotI to release the MoRIC 8 C-terminal GFP-tagging fragment, which was used to insert the NotI site of pCB1532, which contains the ILV1 allele conferring resistance to sulfonylurea (Sweigard et al. 1997) to give RIC-GFP. The resulting plasmid, RIC-GFP, was used to transform into Amoric8 mutant Q-10 and LY-130, respectively. Transformants carrying a single insertion were selected and complementation of the $\Delta$ moric 8 mutant phenotypes were checked. GFP fluorescence was observed using a Leica TCS SP5 inverted confocal laser scanning microscope (Leica, Wetzlar, Germany).

\section{Phenotypic analysis.}

Vegetative growth was assessed by measurement of colony diameter on plate cultures of $M$. oryzae grown on CM (Talbot et al. 1993). Conidial development was assessed by harvesting conidia from the surface of 10-day-old plate cultures and by determining the concentration of the resulting conidial suspension using a hemacytometer. Appressorium development was assessed by allowing conidia at a concentration of $5 \times 10^{4}$ conidia $\mathrm{ml}^{-1}$ to germinate on hydrophobic GelBond films or onion epidermis and incubating them in a humid environment at $25^{\circ} \mathrm{C}$. The frequency of conidial germination and appressorium formation was determined at different time intervals. Each test was repeated three times. Fertility assays were carried out by pairing Guy11 (MAT1-2), LY-130, or moric8 mutants with standard tester strain TH3 (MAT1-1) on oatmeal agar plates. The plates were incubated at $25^{\circ} \mathrm{C}$ with a 12 -h photo-phase until the colonies contacted each other, and then placed under continuous white fluorescent light at $18^{\circ} \mathrm{C}$ for 3 to 4 weeks. The junctions between the mated individuals were examined for the capacity to form perithecia. The mating test was repeated three times.

For the assays of appressorium formation induced by chemicals, conidia from 10-day-old CM plate cultures were resuspended to $5 \times 10^{4}$ conidia $\mathrm{ml}^{-1}$ in sterile distilled water. Exogenous cAMP, IBMX, and Diol were added to conidial suspensions at a final concentration of $10 \mathrm{mM}, 2.5 \mathrm{mM}$, and 1 $\mu \mathrm{M}$, respectively. The events of appressorium formation were assessed under a microscope after incubating them in a humid chamber for $24 \mathrm{~h}$ at $25^{\circ} \mathrm{C}$. More than 300 spores were counted for each treatment. Each test was repeated three times.

\section{Cuticle penetration and plant infection assays.}

Preparation of sterile onion epidermal strips was carried out by the procedure of Chida and Sisler (1987). For penetration assays, $M$. oryzae conidia were harvested from 10-day-old cultures, dropped on strips of onion epidermis with conidial suspensions of $5 \times 10^{4}$ conidia $\mathrm{ml}^{-1}$, and observed for appressorium formation and elaboration of penetration hyphae at $24 \mathrm{~h}$ by microscopy. The frequency of cuticle penetration was determined by counting the penetration events from 300 appressoria. The experiments were repeated three times. Plant infection assays were carried out using cut-leaf assays or seedling sprayinoculation methods described previously (Talbot et al. 1993; Z. Y. Wang et al. 2007). The cut-leaf assay was performed as follows. Leaf fragments were cut from 10-day-old barley cv. Golden Promise or 14-day-old rice cv. CO-39 seedlings, which are highly susceptible toward $M$. oryzae, and placed in plastic plates containing wetted filters. Droplets $(10 \mu \mathrm{l})$ of conidial suspensions at $1 \times 10^{5}$ conidia $\mathrm{ml}^{-1}$ from 10-day-old CM cultures at $25^{\circ} \mathrm{C}$ were placed carefully on leaf sections. The plates were incubated in a cycle of $12 \mathrm{~h}$ of light and $12 \mathrm{~h}$ of darkness at $25^{\circ} \mathrm{C}$. Disease lesions were examined after 5 days of incubation. Wounded rice leaves were prepared by removing the surface cuticle by abrasion with an emery board. Rice seedling infections were carried out as described previously (Talbot et al. 1993). Conidial suspensions were diluted in $0.2 \%$ gelatin to $1 \times 10^{5}$ conidia $\mathrm{ml}^{-1}$ for rice infections using rice cv. CO-39. Conidia were spray inoculated using an artist's airbrush onto 14-day-old plants. Rice seedlings were incubated in plastic bags for $24 \mathrm{~h}$ to maintain high humidity and then transferred to controlled environment chambers at $25^{\circ} \mathrm{C}$ and $90 \%$ relative humidity with illumination and 14-h light periods. Plants were incubated for 5 days for disease symptom development.

\section{qRT-PCR.}

Total RNA was extracted from mycelium of $M$. oryzae Guy11, Q-3, Q-10, and Q-8 (Table 1) using SV Total RNA Isolation System (Z3100; Promega) according to the manufacturer's instructions. Total RNA was utilized for synthesis of the first-strand cDNA using the PrimeScript 1st Strand cDNA Synthesis Kit (D6110A; TaKaRa, Tokyo). The resultant cDNA was used as a template for qRT-PCR. qRT-PCR was performed with a SYBR Green Realtime PCR Master Mix Kit (QPK-201; TOYOBO, Osaka, Japan) using an iCycler iQ Multicolor RealTime PCR Detection System (Bio-Rad, Munich, Germany). All qRT-PCR reactions were conducted in duplicate for each cDNA sample and were repeated three times. The primer pairs of RIC-Q-F/RIC-Q-R, MA-Q-F/MA-Q-R, MB-Q-F/MB-Q-R,

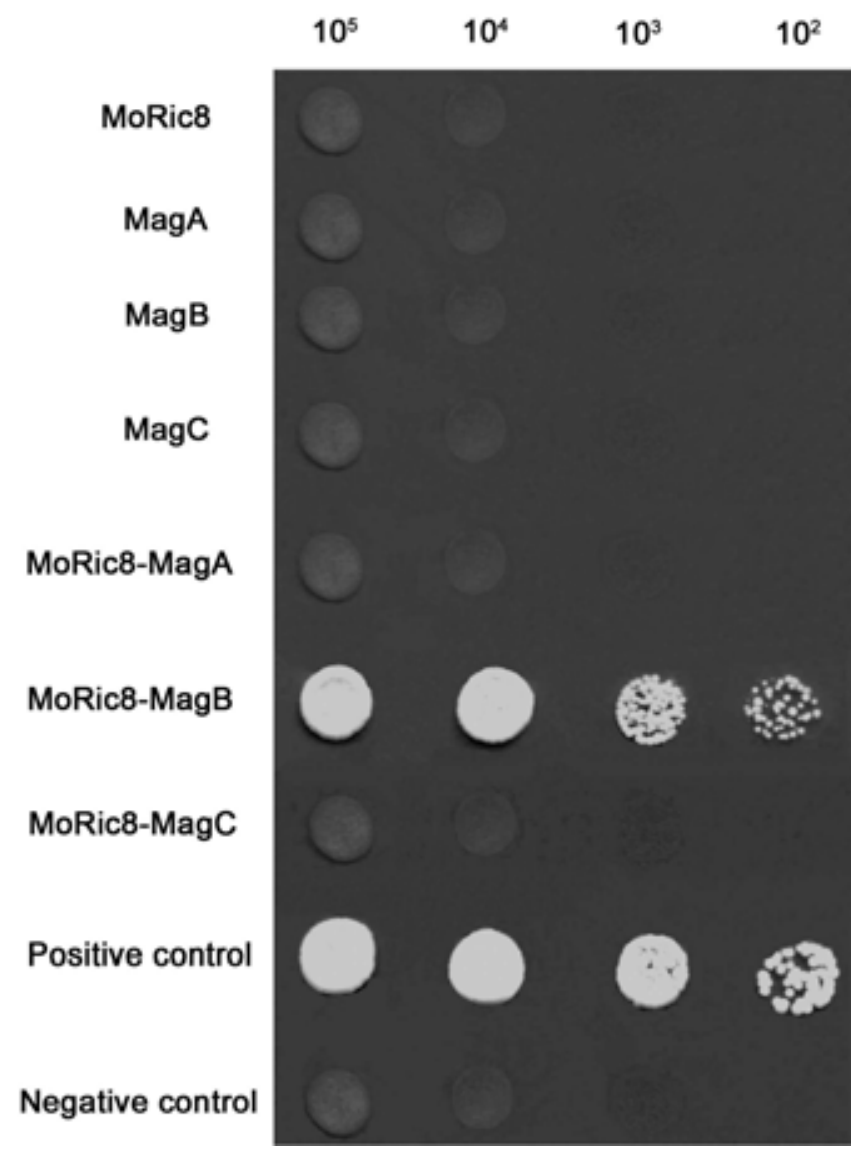

Fig. 8. MoRic8 interacts with the Go subunit MagB. The interactions between MoRic8 and $\mathrm{G}$ proteins were investigated using yeast two-hybrid assay. The constructed prey vector pGADT7-RIC and the bait vector pGBKT7-MAGA, pGBKT7-MAGB, or pGBKT7-MAGC were cotransformed into yeast strain $\mathrm{AH} 109$. The $\mathrm{Leu}^{+}$and $\mathrm{Trp}^{+}$yeast transformants were assayed for growth on SD-Trp-Leu-His-Ade medium at specified concentrations $1 \times 10^{5}, 1 \times 10^{4}, 1 \times 10^{3}$, and $1 \times 10^{2}$ cells per each $10-\mu 1$ droplet. 
MC-Q-F/MC-Q-R, MB1-Q-F/MB1-Q-R, and MG1-Q-F/MG1$\mathrm{Q}-\mathrm{R}$ (Table 2) were used to determine the relative expression of MoRIC8 (MGG_140086.6), MAGA (MGG_01818.6), $M A G B$ (MGG_00365.6), MAGC (MGG_04204.6), MGB1 (MGG_05201.6), and MGG1 (MGG_10193.6), respectively. The $M$. oryzae $\beta$-tubulin gene (MGG_00604.6) amplified with the primer pairs of Tub-F/Tub-R was used as an endogenous control. The abundance of the gene transcripts was calculated relative to this control using the $2^{-\Delta \Delta \mathrm{CT}}$ method (Livak and Schmittgen 2001).

\section{Y2H assay.}

Y2H assay was carried out as the description of BD Matchmaker Library Construction \& Screening Kits (Clontech, Palo Alto, CA, U.S.A.). The full-length cDNA of MoRIC8 was amplified with the primer pair RIC-E-F/RIC-E-R (Table 2) and cloned into pGADT7 as the prey vector pGADT7-RIC. The full-length cDNAs of $M A G A, M A G B$, and $M A G C$ were amplified with the primer pairs MAGA-Nd-F/MAGA-Ps-R, MAGBE-F/MAGB-B-R, and MAGC-E-F/MAGC-B-R (Table 2), respectively, and were cloned into pGBKT7 as the bait vectors pGBKT7-MAGA, pGBKT7-MAGB, and pGBKT7-MAGC. The resulting pGADT7-RIC and pGBKT7-MAGA, pGBKT7MAGB, or pGBKT7-MAGC, were cotransformed into yeast strain $\mathrm{AH} 109$. The $\mathrm{Leu}^{+}$and $\mathrm{Trp}^{+}$yeast transformants were isolated and assayed for growth on SD-Trp-Leu-His-Ade medium at specified concentrations of $1 \times 10^{5}, 1 \times 10^{4}, 1 \times 10^{3}$, and $1 \times$ $10^{2}$ cells per each $10-\mu$ d droplet. Yeast stains for positive and negative controls were from the Kit.

\section{ACKNOWLEDGMENTS}

This manuscript was selected as a feature article through the APS/CSPP Cooperative Agreement. This work was supported by National Key Basic Research and Development Program (2006CB101901), the Natural Science Foundation of China (Nos. 30570054 and 30670073), Commonweal Specialized Research Fund of China Agriculture (200803008), Ministry of Agriculture of China (2006-G61), and Ministry of Science and Technology of Zhejiang Province (2008R10036) to Z.-Y. Wang.

\section{LITERATURE CITED}

Adachi, K., and Hamer, J. E. 1998. Divergent cAMP signaling pathways regulate growth and pathogenesis in the rice blast fungus Magnaporthe grisea. Plant Cell 10:1361-1373.

Afshar, K., Willard, F. S., Colombo, K., Johnston, C. A., McCudden, C. R., Siderovski, D. P., and Gonczy, P. 2004. RIC-8 is required for GPR1/2-dependent $\mathrm{G} \alpha$ function during asymmetric division of $C$. elegans embryos. Cell 119:219-230.

Bundock, P., den Dulk-Ras, A., Beijersbergen, A., and Hooykaas, P. J. 1995. Trans-kingdom T-DNA transfer from Agrobacterium tumefaciens to Saccharomyces cerevisiae. EMBO (Eur. Mol. Biol. Organ.) J. 14:3206-3214.

Caracuel-Rios, Z., and Talbot, N. J. 2007. Cellular differentiation and host invasion by the rice blast fungus Magnaporthe grisea. Curr. Opin. Microbiol. 10:339-345.

Carroll, A. M., Sweigard, J. A., and Valent, B. 1994. Improved vectors for selecting resistance to hygromycin. Fungal Genet. Newsl. 41:22.

Chida, T., and Sisler, H. D. 1987. Restoration of appressorial penetration ability by melanin precursors in Pyricularia oryzae treated with antipenetrants and in melanin-deficient mutants. J. Pestic. Sci. 12:49-55.

Chiu, W. L., Niwa, Y., Zeng, W., Hirano, T., Kobayashi, H., and Sheen, J. 1996. Engineered GFP as a vital reporter in plants. Curr. Biol. 6:325330.

Choi, J., Park, J., Jeon, J., Chi, M. H., Goh, J., Yoo, S. Y., Park, J., Jung, K., Kim, H., Park, S. Y., Rho, H. S., Kim, S., Kim, B. R., Han, S. S., Kang, S., and Lee, Y. H. 2007. Genome-wide analysis of T-DNA integration into the chromosomes of Magnaporthe oryzae. Mol. Microbiol. 66:371-382.

Choi, W. B., and Dean, R. A. 1997. The adenylate cyclase gene MAC1 of Magnaporthe grisea controls appressorium formation and other aspects of growth and development. Plant Cell 9:1973-1983.

De Groot, M. J. A., Bundock P., Hooykaas P. J. J., and Beijersbergen, A.
G. M. 1998. Agrobacterium tumefaciens-mediated transformation of filamentous fungi. Nat. Biotechnol. 16:839-842.

Dean, R. A., Talbot, N. J., Ebbole, D. J., Farman, M. L., Mitchell, T. K., Orbach, M. J., Thon, M., Kulkarni, R., Xu, J. R., Pan, H., Read, N. D., Lee, Y. H., Carbone, I., Brown, D., Oh, Y. Y., Donofrio, N., Jeong, J. S., Soanes, D. M., Djonovic, S., Kolomiets, E., Rehmeyer, C., Li, W., Harding, M., Kim, S., Lebrun, M. H., Bohnert, H., Coughlan, S., Butler, J., Calvo, S., Ma, L. J., Nicol, R., Purcell, S., Nusbaum, C., Galagan, J. E., and Birren, B. W. 2005. The genome sequence of the rice blast fungus Magnaporthe grisea. Nature 434:980-986.

Ebbole, D. J. 2007. Magnaporthe as a model for understanding hostpathogen interactions. Annu. Rev. Phytopathol. 45:437-56.

Fang, E. G., and Dean, R. A. 2000. Site-directed mutagenesis of the magB gene affects growth and development in Magnaporthe grisea. Mol. Plant-Microbe Interact. 13:1214-1227.

Gupta, A., and Chattoo, B. B. 2007. A novel gene MGA1 is required for appressorium formation in Magnaporthe grisea. Fungal Genet. Biol. 44:1157-1169.

Jeon, J., Park, S. Y., Chi, M. H., Choi, J., Park, J., Rho, H. S., Kim, S., Goh, J., Yoo, S., Choi, J., Park, J. Y., Yi, M., Yang, S., Kwon, M. J., Han, S. S., Kim, B. R., Khang, C. H., Park, B., Lim, S. E., Jung, K., Kong, S., Karunakaran, M., Oh, H. S., Kim, H., Kim, S., Park, J., Kang, S., Choi, W. B., Kang, S., and Lee, Y. H. 2007. Genome-wide functional analysis of pathogenicity genes in the rice blast fungus. Nat. Genet. 39:561-565.

Kankanala, P., Czymmek, K., and Valent, B. 2007. Roles for rice membrane dynamics and plasmodesmata during biotrophic invasion by the blast fungus. Plant Cell 19:706-724.

Leung, H., Borromeo, E. S., Bernardo, M. A., and Notteghem, J. L. 1988. Genetic analysis of virulence in the rice blast fungus Magnaporthe grisea. Phytopathology 78:1227-1233.

Li, L., Wright, S. J., Krystofova, S., Park, G., and Borkovich, K. A. 2007. Heterotrimeric $G$ protein signaling in filamentous fungi. Annu. Rev. Microbiol. 61:423-452.

Liu, H., Suresh, A., Willard, F. S., Siderovski, D. P., Lu, S., and Naqvi, N. I. 2007. Rgs1 regulates multiple Galpha subunits in Magnaporthe pathogenesis, asexual growth and thigmotropism. EMBO (Eur. Mol. Biol. Organ.) J. 26:690-700.

Liu, S. H., and Dean, R. A. 1997. G protein alpha subunit genes control growth, development, and pathogenicity of Magnaporthe grisea. Mol. Plant-Microbe Interact. 10:1075-1086.

Liu, Y. G., and Chen, Y. 2007. High-efficiency thermal asymmetric interlaced PCR for amplification of unknown flanking sequences. Biotechniques 43:649-650.

Livak, K. J., and Schmittgen, T. D. 2001. Analysis of relative gene expression data using real-time quantitative PCR and the $2^{-\Delta \Lambda C T}$ method. Methods 25:402-408

Malik, S., Ghosh, M., Bonacci, T. M., Tall, G. G., and Smrcka, A. V. 2005. Ric- 8 enhances $G$ protein $\beta \gamma$-dependent signaling in response to $\beta \gamma$ binding peptides in intact cells. Mol. Pharmacol. 68:129-136.

Meng, Y., Patel, G., Heist, M., Betts, M. F., Tucker, S. L., Galadima, N., Donofrio, N. M., Brown, D., Mitchell, T. K., Li, L., Xu, J. R., Orbach, M., Thon, M., Dean, R. A., and Farman, M. L. 2007. A systematic analysis of T-DNA insertion events in Magnaporthe oryzae. Fungal Genet. Biol. 44:1050-1064.

Miller, K. G., Alfonso, A., Nguyen, M., Crowell, J. A., Johnson, C. D., and Rand, J. B. 1996. A genetic selection for Caenorhabditis elegans synaptic transmission mutants. Proc. Natl. Acad. Sci. U.S.A. 93:12593-12598.

Miller, K. G., Emerson, M. D., McManus, J. R., and Rand, J. B. 2000. RIC-8 (Synembryn): a novel conserved protein that is required for $\mathrm{G}(\mathrm{q})$ alpha signaling in the C. elegans nervous system. Neuron 27:289-299.

Mitchell, T. K., and Dean, R. A. 1995. The cAMP-dependent protein kinase catalytic subunit is required for appressorium formation and pathogenesis by the rice blast pathogen Magnaporthe grisea. Plant Cell 7:1869-1878.

Mullins, E. D., Chen, X., Romaine, P., Raina, R., Geiser, D. M., and Kang, S. 2001. Agrobacterium-mediated transformation of Fusarium oxysporum: an efficient tool for insertional mutagenesis and gene transfer. Phytopathology 91:173-180.

Neves, S. R., Ram, P. T., and Iyengar, R. 2002. G protein pathways. Science 296:1636-1639.

Nishimura, M., Park, G., and Xu, J. R. 2003. The G-beta subunit MGB1 is involved in regulating multiple steps of infection-related morphogenesis in Magnaporthe grisea. Mol. Microbiol. 50:231-243.

Park, G., Xue, C., Zhao, X., Kim, Y., Orbach, M., and Xu, J. R. 2006. Multiple upstream signals converge on the adaptor protein Mst50 in Magnaporthe grisea. Plant Cell 18:2822-2835.

Rho, H. S., Kang, S., and Lee, Y. H. 2001. Agrobacterium tumefaciens mediated transformation of the plant pathogenic fungus, Magnaporthe grisea. Mol. Cells 12:407-411. 
Sambrook, J., Fritsch, E. F., and Maniatis, T. 1989. Molecular Cloning: A Laboratory Manual. Cold Spring Harbor Laboratory Press, Cold Spring Harbor, NY, U.S.A.

Schacht, P. C., and Borkovich, K. A. 2009. Studying the non receptor GEF RIC8 using inhibitors and suppressors in Neurospora crassa. Fungal Genet. Rep. 56 (Suppl.):241

Siderovski, D. P., and Willard, F. S. 2005. The GAPs, GEFs, and GDIs of heterotrimeric G-protein alpha subunits. Int. J. Biol. Sci. 1:51-66.

Sweigard, J. A., Carroll, A. M., Farrall, L., and Valent, B. 1997. A series of vectors for fungal transformation. Fungal Genet. Newsl. 44:52-53.

Talbot, N. J. 2003. On the trail of a cereal killer. Annu. Rev. Microbiol. 57:177-202.

Talbot, N. J., Ebbole, D. J., and Hamer, J. E. 1993. Identification and characterization of $M P G 1$, a gene involved in pathogenicity from the rice blast fungus Magnaporthe grisea. Plant Cell 5:1575-1590.

Tall, G. G., Krumins, A. M., and Gilman, A. G. 2003. Mammalian Ric-8A (synembryn) is a heterotrimeric Galpha protein guanine nucleotide exchange factor. J. Biol. Chem. 278:8356-8362.

Wang, H., Ng, K. H., Qian, H., Siderovski, D. P., Chia, W., and Yu, F. 2005. Ric-8 controls Drosophila neural progenitor asymmetric division by regulating heterotrimeric G proteins. Nat. Cell Biol. 7:1091-1098

Wang, S. C., Lai, H. L., Chiu, Y. T., Ou, R., Huang, C. L., and Chern, Y. 2007. Regulation of type V adenylate cyclase by Ric8a, a guanine nucleotide exchange factor. Biochem. J. 406:383-388.
Wang, S. C., Lin, J. T., and Chern, Y. 2009. Novel regulation of adenyly cyclases by direct protein-protein interactions: insights from snapin and Ric8a. Neurosignals 17:169-180.

Wang, Z. Y., Soanes, D. M., Kershaw, M. J., and Talbot, N. J. 2007. Functional analysis of lipid metabolism in Magnaporthe grisea reveals a role for peroxisomal fatty acid b-oxidation during appressorium-mediated plant infection. Mol. Plant-Microbe Interact. 20:475-491.

Wilson, R. A., and Talbot, N. J. 2009. Under pressure: investigating the biology of plant infection by Magnaporthe oryzae. Nat. Rev. Microbiol. 7:185-195.

Wright, S., Krystofova, S., and Borkovich, K. A. 2009. RIC8 regulates polar growth in Neurospora crassa. Fungal Genet. Rep. 56 (Suppl.):242

Xu, J. R., and Hamer, J. E. 1996. MAP kinase and cAMP signaling regulate infection structure formation and pathogenic growth in the rice blast fungus Magnaporthe grisea. Genes Dev. 10:2696-2706.

Xu, J. R., Urban, M., Sweigard, J. A., and Hamer, J. E. 1997. The CPKA gene of Magnaporthe grisea is essential for appressorial penetration. Mol. Plant-Microbe Interact. 10:187-194.

Zhao, X., and Xu, J. R. 2007. A highly conserved MAPK-docking site in Mst7 is essential for Pmk1 activation in Magnaporthe grisea. Mol. Microbiol. 63:881-894.

Zhao, X., Kim, Y., Park, G., and Xu, J. R. 2005. A mitogen-activated protein kinase cascade regulating infection-related morphogenesis in $\mathrm{Mag}$ naporthe grisea. Plant Cell 17:1317-1329. 